JOURNAL OF THE

AMERICAN MATHEMATICAL SOCIETY

Volume 16, Number 4, Pages 857-885

S 0894-0347(03)00432-6

Article electronically published on June 19, 2003

\title{
BILLIARDS AND TEICHMÜLLER CURVES ON HILBERT MODULAR SURFACES
}

\author{
CURTIS T. MCMULLEN
}

\section{INTRODUCTION}

As a projective variety, the moduli space $\mathcal{M}_{g}$ of Riemann surfaces of genus $g$ is swept out by algebraic curves. Only rarely, however, are these curves isometrically embedded for the Teichmüller metric.

In this paper we address the classification of isometrically embedded curves in $\mathcal{M}_{2}$. In addition to the curves inherited from $\mathcal{M}_{1}$, we find an infinite collection lying on Hilbert modular surfaces parameterizing Jacobians with real multiplication. Explicit examples, constructed from L-shaped polygons, give billiard tables with optimal dynamical properties.

Complex geodesics. Let $f: \mathbb{H} \rightarrow \mathcal{M}_{g}$ be a holomorphic map from the hyperbolic plane of constant curvature -4 to the moduli space of Riemann surfaces of genus $g$ with the Teichmüller metric.

A theorem of Royden asserts that $f$ is either an isometry or a contraction. When $f$ is an isometry, it parameterizes a complex geodesic in moduli space. A typical complex geodesic is dense and uniformly distributed.

On rare occasions, a complex geodesic may cover an algebraic curve in moduli space. Then the stabilizer $\Gamma$ of $f$ is a lattice in Aut $(\mathbb{H})$; passing to the quotient, we obtain a Teichmüller curve

$$
f: V=\mathbb{H} / \Gamma \rightarrow \mathcal{M}_{g}
$$

Every complex geodesic yields a Teichmüller curve when $g=1$, since $\mathcal{M}_{1}$ is itself a curve. By taking branched coverings of tori, one obtains Teichmüller curves in $\mathcal{M}_{g}$ for all $g$.

A Teichmüller curve is primitive if it does not arise from a curve in a moduli space of lower genus via a branched covering construction. To date only a finite number of primitive Teichmüller curves have been found in each $\mathcal{M}_{g}$.

In this paper we undertake the classification of Teichmüller curves in genus two and obtain an infinite family of primitive examples.

Jacobians. We approach Teichmüller curves via the families of Jacobians they determine.

Received by the editors April 8, 2002.

2000 Mathematics Subject Classification. Primary 32G15; Secondary 37D50, 11F41, 14 G35.

Research supported in part by the NSF. 
Every complex geodesic is generated by a pair $(X, q)$, where $q$ is a quadratic differential on $X \in \mathcal{M}_{g}$. We concentrate on the case where $q=\omega^{2}$ is the square of a holomorphic 1-form; then we have (\$4, Kra]):

Theorem 1.1. For any complex geodesic $f: \mathbb{H} \rightarrow \mathcal{M}_{g}$ generated by $\left(X, \omega^{2}\right)$, the composed map

$$
\mathbb{H} \stackrel{f}{\rightarrow} \mathcal{M}_{g} \rightarrow \mathcal{A}_{g}
$$

is an isometry for the Kobayashi metric.

Here $\mathcal{A}_{g}$ denotes the moduli space of principally polarized Abelian varieties, and the map $\mathcal{M}_{g} \rightarrow \mathcal{A}_{g}$ sends a curve to its Jacobian.

This result suggests the classification of Teichmüller curves will benefit from an analysis of isometrically embedded curves on $\mathcal{A}_{g}$, which unlike $\mathcal{M}_{g}$ is covered by a symmetric space.

Hilbert modular surfaces. Recall that $A \in \mathcal{A}_{g}$ admits real multiplication if $\operatorname{End}(A) \otimes \mathbb{Q}$ contains a totally real field with $\operatorname{deg}(K / \mathbb{Q})=g$. The space of Abelian varieties with a given action of an order in $K$ is parameterized by a Hilbert modular variety.

In $\$ 7$ we show that Teichmüller curves and real multiplication are closely connected in the case of genus two.

Theorem 1.2. Let $f: V \rightarrow \mathcal{M}_{2}$ be a primitive Teichmüller curve generated by $\left(X, \omega^{2}\right)$. Then the projection of $V$ to $\mathcal{A}_{2}$ lies in the locus of real multiplication, yielding a commutative diagram

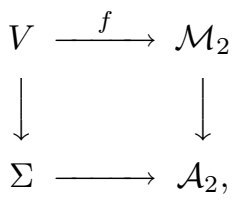

where $\Sigma$ is a Hilbert modular surface.

Weierstrass forms. In $\$ 8$ we give a synthetic description of an infinite collection of Teichmüller curves in $\mathcal{M}_{2}$. A Weierstrass form is a holomorphic 1 -form whose zero divisor is concentrated at a single point.

Theorem 1.3. The locus

$$
\begin{gathered}
\mathcal{W}_{2}=\left\{X \in \mathcal{M}_{2}: \operatorname{Jac}(X)\right. \text { admits real multiplication with } \\
\text { a Weierstrass eigenform }\}
\end{gathered}
$$

is a union of infinitely many primitive Teichmüller curves.

Let $\operatorname{SL}(X, \omega)$ denote the linear parts of automorphisms of $X$ preserving the oriented real-affine structure determined by $\omega$. The pair $\left(X, \omega^{2}\right)$ generates a Teichmüller curve iff $\operatorname{SL}(X, \omega)$ is a lattice. The results above imply:

Corollary 1.4. In genus two, a Weierstrass form generates a primitive Teichmüller curve if and only if there is a $T \in \mathrm{SL}(X, \omega)$ with irrational trace.

This Corollary yields a complete classification of the finite-volume $\mathrm{SL}_{2}(\mathbb{R})$-orbits in $\Omega \mathcal{M}_{2}(2)$, the Weierstrass stratum of the bundle of all holomorphic 1 -forms over $\mathcal{M}_{2}$. 
Corresponding results break down in higher genus; for example, the locus of eigenforms for real multiplication $\mathcal{E}_{g} \subset \Omega \mathcal{M}_{g}$ is $\mathrm{SL}_{2}(\mathbb{R})$-invariant for $g=2$ but not for $g=3$ (see \$7).

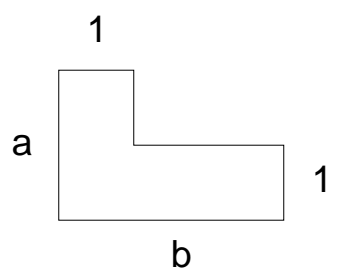

Figure 1. The L-shaped polygon $P(a, b)$.

Billiards. Using L-shaped polygons we obtain explicit examples of Teichmüller curves as above, as well as billiard tables with optimal dynamical properties.

Let $P \subset \mathbb{C}$ be a polygon whose angles are rational multiplies of $\pi$. Via an unfolding construction, the polygon $P$ determines a holomorphic 1-form $\omega$ on a compact Riemann surface $X$. Billiard paths on $P$ transform into geodesics on $X$ for the singular flat metric $|\omega|$.

If $(X, \omega)$ generates a Teichmüller curve, then $P$ is a lattice polygon. Veech showed that the billiard flow in a lattice polygon exhibits sharp dynamical features; for example, every billiard path is periodic or uniformly distributed, and the number of closed trajectory types of length $\leq L$ is asymptotic to $c(P) L^{2}$.

Using the results above, we obtain a new infinite family of lattice polygons of the type $P(a, b)$ shown in Figure 1 .

Theorem 1.5. The L-shaped billiard table $P(a, b)$ is a lattice polygon iff $a$ and $b$ are rational or

$$
a=x+z \sqrt{d} \quad \text { and } \quad b=y+z \sqrt{d}
$$

for some $x, y, z \in \mathbb{Q}$ with $x+y=1$ and $d \geq 0$ in $\mathbb{Z}$.

We also determine the uniformizations of the Teichmüller curves $V=\mathbb{H} / \Gamma$ generated by $P(a, a)$ explicitly, for many values of $a$. In particular we find the first examples of Teichmüller curves such that $\Gamma$ is not commensurable to a triangle group. See $\$ 9$

In the final section we observe that a primitive Teichmüller curve $V=\mathbb{H} / \Gamma$ on a Hilbert modular surface $\Sigma$ is covered by the graph of a remarkable holomorphic map $F: \mathbb{H} \rightarrow \mathbb{H}$, intertwining the action of the discrete group $\Gamma \subset \mathrm{SL}_{2}(K)$ with its indiscrete Galois conjugate $\Gamma^{\prime}$.

Notes and references. Veech gave the first examples of Teichmüller curves beyond those arising from tori, by showing that the regular polygons and the isosceles triangles that tile them are lattice polygons [V1], V3]. In these cases $\mathrm{SL}(X, \omega)$ is commensurable to the $(2, n, \infty)$ triangular group. Additional triangular lattice polygons appear in $[\mathrm{Wa}]$ and $[\mathrm{KS}]$, and the classification of acute lattice triangles, begun in [KS], is completed in [Pu]; see also [V3], [Vo], GJ]. General references for billiards include $\mathrm{Tab}$ and $\mathrm{MT}$. The application of Teichmüller theory to billiards dates from the work of Kerckhoff, Masur and Smillie [KMS]. 
Another classification of Teichmüller curves in $\mathcal{M}_{2}$, using different methods (such as the Kenyon-Smillie invariant [ $\mathrm{KS}]$ ), is announced by K. Calta in [Ca].

To set the stage for the discussion that follows, we have included background on the moduli space of holomorphic 1-forms and on Hilbert modular varieties, as well as the trace field of the Fuchsian group uniformizing a Teichmüller curve.

\section{TeICHMÜLLER CURVES}

In this section we introduce Teichmüller curves and recall their connection to the dynamics of foliations, established in [V1].

Teichmüller space. Let $Z_{g}$ denote an oriented smooth surface of genus $g$. Let $\mathcal{T}_{g}$ denote the Teichmüller space of marked Riemann surfaces $X$ of genus $g$, each equipped with an orientation-preserving homeomorphism $h: Z_{g} \rightarrow X$ up to isotopy. For $g>1, \mathcal{T}_{g}$ can be realized as a bounded domain of holomorphy in $\mathbb{C}^{3 g-3}$.

The Teichmüller metric is defined by

$$
d(X, Y)=\frac{1}{2} \inf _{\phi} \log K(\phi: X \rightarrow Y),
$$

where $\phi$ ranges over all quasiconformal maps from $X$ to $Y$ compatible with their markings, and $K(\phi)$ is the dilatation of $\phi$. The Teichmüller metric coincides with the Kobayashi metric; in genus 1 , it gives the metric of curvature -4 on $\mathcal{T}_{1} \cong \mathbb{H}$.

The moduli space $\mathcal{M}_{g}=\mathcal{T}_{g} / \operatorname{Mod}\left(Z_{g}\right)$ is the quotient of $\mathcal{T}_{g}$ by the action of the mapping-class group of $Z_{g}$; it is a complex orbifold as well as an algebraic variety.

Complex geodesics. Let $Q(X)$ denote the space of holomorphic quadratic differentials on $X \in \mathcal{M}_{g}$. We have $\operatorname{dim} Q(X)=3 g-3$ for $g>1$.

A pair $(X, q)$ with $q \in Q(X), q \neq 0$, generates a holomorphic embedding $\widetilde{f}: \mathbb{H} \rightarrow$ $\mathcal{T}_{g}$ which is an isometry for the Kobayashi metrics on domain and range. Passing to the quotient by the action of the mapping-class group, we obtain a complex geodesic

$$
f: \mathbb{H} \rightarrow \mathcal{M}_{g}
$$

We can regard $f$ as the complexification of the real geodesic $f\left(i e^{2 s}\right), s \in \mathbb{R}$.

The surface $X_{t}=\widetilde{f}(t)$ is characterized by the property that the extremal quasiconformal map $\phi_{t}: X \rightarrow X_{t}$ has complex dilatation

$$
\mu_{t}=\frac{d \phi_{t} / d \bar{z}}{d \phi_{t} / d z}=\left(\frac{i-t}{i+t}\right) \cdot \frac{\bar{q}}{|q|} .
$$

Note that $\mu_{t}$ maps $\mathbb{H}$ to the unit disk in the complex line of Beltrami differentials spanned by $\bar{q} /|q|$.

Teichmüller curves. Let $f: \mathbb{H} \rightarrow \mathcal{M}_{g}$ be a complex geodesic generated by $(X, q)$. Then $f$ factors through the quotient space $V=\mathbb{H} / \operatorname{Stab}(f)$, where

$$
\operatorname{Stab}(f)=\{A \in \operatorname{Aut}(\mathbb{H}): f(A \cdot t)=f(t) \forall t\} .
$$

If $V$ has finite hyperbolic area (that is, if $\operatorname{Stab}(f)$ is a lattice), then the quotient map

$$
f: V \rightarrow \mathcal{M}_{g}
$$

is a Teichmüller curve on $\mathcal{M}_{g}$. The map $f$ is proper and generically injective. Its image $V^{\prime}=f(V) \subset \mathcal{M}_{g}$ is an algebraic curve, whose normalization is $V$.

We will informally refer to $V^{\prime}$ itself as a Teichmüller curve, its normalization by $f$ being implicitly understood. 
Dynamics of foliations. A quadratic differential $q \neq 0$ determines a foliation $\mathcal{F}(q)$ of $X$, tangent to the vectors with $q(v)>0$ and with singularities at the zeros of $q$. This foliation has a tight dynamical structure when $(X, q)$ generates a Teichmüller curve.

To describe this structure, define a leaf of $\mathcal{F}(q)$ to be a maximal connected immersed 1-manifold $L \subset X$ with $T L \subset T \mathcal{F}(q)$ and with no zeros of $q$ in its interior. A compact leaf is either a loop $L \cong S^{1}$ or an interval $L \cong[0,1]$ joining a pair of zeros (a saddle connection). A noncompact leaf is an immersed copy of $\mathbb{R}$ or of $[0, \infty)$; in the latter case, $L$ is a ray emanating from a zero of $q$.

A noncompact leaf $L$ is uniformly distributed on $X$ if, for any immersion $\gamma$ : $[0, \infty) \rightarrow L$ moving at constant speed in the metric $|q|$, and any open set $U \subset X$, we have

$$
\lim _{T \rightarrow \infty} \frac{|\{t \in[0, T]: \gamma(t) \in U\}|}{T}=\frac{\int_{U}|q|}{\int_{X}|q|} .
$$

A simple closed geodesic for the flat metric $|q|$ is an embedded loop $L \subset X$ that coincides with a leaf of $\mathcal{F}\left(e^{i \theta} q\right)$ for some $\theta$. Its length is given by $\int_{L}|q|^{1 / 2}$. Let $N(T, q)$ denote the number of isotopy classes of loops on $X$ represented by simple closed geodesics of length $\leq T$.

We can now state a central result from [V1, 2.11 and 3.10].

Theorem 2.1 (Veech). Let $(X, q)$ generate a Teichmüller curve. Then for any $\theta$ either:

(1) all leaves of $\mathcal{F}\left(e^{i \theta} q\right)$ are compact, or

(2) all leaves of $\mathcal{F}\left(e^{i \theta} q\right)$ are noncompact and uniformly distributed on $X$.

Moreover the number of isotopy classes of simple closed geodesics satisfies $N(T, q) \sim$ $c T^{2}$ for some constant $c(X, q)>0$.

To put the dichotomy of Theorem 2.1 in context, we note that for any $q \neq 0$ in $Q(X)$ :

(1) above holds for at most a countable set of $\theta$, while

(2) holds for almost all $\theta$ in the sense of measure KMS, Mas3.

We also have $0<a T^{2}<N(T, q)<b T^{2}$ for some $a, b$ [Mas2].

On the other hand, it is easy to give examples of quadratic differentials such that $\mathcal{F}(q)$ has both compact and noncompact leaves; in general, neither condition (1) nor (2) holds. There are also examples where $\mathcal{F}(q)$ has dense leaves that are not uniformly distributed; see [CFS, Chapter 5.4], [MT §3]. (The former example is given as an interval exchange transformation.)

Using the existence of a saddle connection, one finds [V1]:

Proposition 2.2. If $f: V \rightarrow \mathcal{M}_{g}$ is a Teichmüller curve, then $V$ has at least one cusp.

Remarks. The proof of Theorem [2.1]in [V1] has a gap (Prop. B on p. 561 is false) that can easily be bridged using the criterion for nonunique ergodicity given in Mas3. See [GJ] for more on the constant $c(X, q)$.

\section{HOLOMORPHIC 1-FORMS}

In the sequel we will focus on Teichmüller curves generated by the squares of holomorphic 1-forms. This section summarizes the connection between complex 
geodesics and the action of $\mathrm{SL}_{2}(\mathbb{R})$ on the space of 1 -forms. It also presents the construction of Teichmüller curves via branched coverings.

Affine maps. A map $B: \mathbb{C} \rightarrow \mathbb{C}$ is real-affine if, after identifying $\mathbb{C}$ with $\mathbb{R}^{2}$, it has the form $B(v)=A v+b$ with $A \in \mathrm{GL}_{2}(\mathbb{R})$ and $b \in \mathbb{R}^{2}$.

Given $X \in \mathcal{M}_{g}$, let $\Omega(X)$ denote the $g$-dimensional space of holomorphic 1-forms on $X$. A nonzero form $\omega \in \Omega(X)$ provides, away from its zeros, a flat metric $|\omega|$ on $X$ and local charts $\int \omega: U \rightarrow \mathbb{C}$ whose transition functions are translations.

Given a pair of Riemann surfaces $(X, \omega)$ and $(Y, \eta)$, each equipped with a nonzero holomorphic 1-form, we say a homeomorphism $\phi:(X, \omega) \rightarrow(Y, \eta)$ is affine if the following equivalent conditions are satisfied.

(1) The map $\phi$ has the form of a real-affine map in the charts determined by $\omega$ and $\eta$.

(2) The map $\phi$ is smooth outside the zeros of $\omega$, and the vector space of 1-forms spanned by $(\operatorname{Re} \omega, \operatorname{Im} \omega)$ is the same as the span of $\phi^{*}(\operatorname{Re} \eta, \operatorname{Im} \eta)$.

(3) Given a lift $\widetilde{\phi}: \widetilde{X} \rightarrow \widetilde{Y}$ of $\phi$ to the universal covers of $X$ and $Y$, there is a real-affine map $B$ such that the diagram

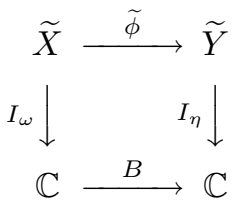

commutes. Here $I_{\omega}(q)=\int_{p}^{q} \widetilde{\omega}$ is obtained by integrating the lift of $\omega$, and similarly for $I_{\eta}$.

We denote the linear part of an affine map by $D \phi \in \mathrm{GL}_{2}(\mathbb{R})$; it agrees with the linear part of $B$ in (3.1).

Action of $\mathrm{GL}_{2}^{+}(\mathbb{R})$ on 1-forms. The spaces $\Omega(X)$ assemble to form a vector bundle over $\mathcal{T}_{g}$. Let

$$
\Omega \mathcal{T}_{g} \rightarrow \mathcal{T}_{g}
$$

denote the complement of the zero section. A point $(X, \omega) \in \Omega \mathcal{T}_{g}$ is a marked Riemann surface together with a nonzero holomorphic 1-form.

Let $\mathrm{GL}_{2}^{+}(\mathbb{R}) \subset \mathrm{GL}_{2}(\mathbb{R})$ be the connected component of the identity, defined by $\operatorname{det}(A)>0$.

There is a natural action of $\mathrm{GL}_{2}^{+}(\mathbb{R})$ on $\Omega \mathcal{T}_{g}$, defined as follows. Given $A=$ $\left(\begin{array}{ll}a & b \\ c & d\end{array}\right) \in \mathrm{GL}_{2}^{+}(\mathbb{R})$ and $(X, \omega) \in \Omega \mathcal{T}_{g}$, consider the harmonic 1-form

$$
\eta=\left(\begin{array}{ll}
1 & i
\end{array}\right)\left(\begin{array}{ll}
a & b \\
c & d
\end{array}\right)\left(\begin{array}{l}
\operatorname{Re} \omega \\
\operatorname{Im} \omega
\end{array}\right)
$$

on $X$. There is a unique complex structure with respect to which $\eta$ is holomorphic; its charts yield a new Riemann surface $Y$, and we define

$$
A \cdot(X, \omega)=(Y, \eta)
$$

The natural affine map $\phi:(X, \omega) \rightarrow(Y, \eta)$ with $D \phi=A$, coming from the change of structure, is the extremal quasiconformal map in its isotopy class. The condition $\operatorname{det}(A)>0$ insures that $\phi$ preserves orientation.

The fibers of the projection $\Omega \mathcal{T}_{g} \rightarrow \mathcal{T}_{g}$ are stabilized by the group of similarities, $\mathbb{R}^{*} \cdot \mathrm{SO}_{2}(\mathbb{R}) \subset \mathrm{GL}_{2}^{+}(\mathbb{R})$. The action of $\mathrm{GL}_{2}^{+}(\mathbb{R})$ commutes with the action of $\operatorname{Mod}\left(Z_{g}\right)$, so it passes to an action on the quotient bundle $\Omega \mathcal{M}_{g} \rightarrow \mathcal{M}_{g}$. 
Periods and strata. Using (3.2), it is easy to see how the periods of 1-forms vary. Namely if $(Y, \eta)=A \cdot(X, \omega)$ in $\Omega \mathcal{T}_{g}$, then

$$
\int_{c} \eta=A\left(\int_{c} \omega\right)
$$

for any cycle $c \in H_{1}\left(Z_{g}, \mathbb{R}\right)$. Here the markings are used to identify $H_{1}\left(Z_{g}\right)$ with $H_{1}(X)$ and $H_{1}(Y)$, and we regard $A$ as acting linearly on $\mathbb{C}=\mathbb{R}^{2}$.

The zeros of $\omega$ determine a divisor $(\omega)=\sum n_{i} P_{i}$ on $X$, and hence an (unordered) partition $n=\left(n_{1}, \ldots, n_{r}\right)$ of $2 g-2$. The space $\Omega \mathcal{T}_{g}$ breaks up into strata

$$
\Omega \mathcal{T}_{g}=\bigcup \Omega \mathcal{T}_{g}\left(n_{1}, \ldots, n_{r}\right)
$$

along which the partition $\left(n_{i}\right)$ is fixed. Each stratum is invariant under $\mathrm{GL}_{2}^{+}(\mathbb{R})$ and $\operatorname{Mod}(S)$.

It is a general principle that a geometric structure is locally determined by its holonomy. In the case at hand, the holonomy can be described by fixing a finite set $D_{r} \subset Z_{g}$ with $\left|D_{r}\right|=r$ and refining the marking of $(X, \omega) \in \Omega \mathcal{T}_{g}\left(n_{1}, \ldots, n_{r}\right)$ so that $\phi$ sends $D_{r}$ to the zeros of $(\omega)$. The marking allows one to transfer the closed form $\omega$ to a relative cohomology class in $H^{1}\left(Z_{g}, D_{r} ; \mathbb{C}\right)$. By [V2] we then have:

Theorem 3.1. Each stratum $\Omega \mathcal{T}_{g}(n)$ is a complex manifold, and the period mapping $\Omega \mathcal{T}_{g}\left(n_{1}, \ldots, n_{r}\right) \rightarrow H^{1}\left(Z_{g}, D_{r} ; \mathbb{C}\right)$ is a holomorphic local homeomorphism.

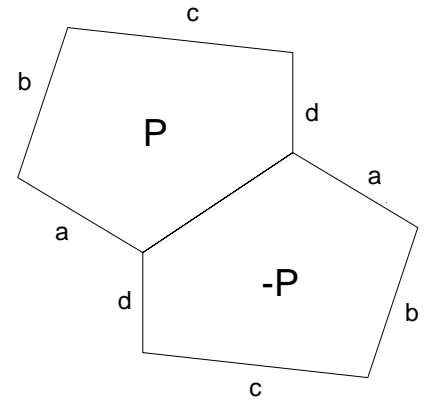

Figure 2. A pentagon determines a surface of genus two.

Pentagons. As an example, we describe part of the stratum $\Omega \mathcal{T}_{2}(2)$. Let $P \subset \mathbb{C}$ be a pentagon. Translate $P$ so $z=0$ is the midpoint of an edge; then $P \cup(-P)$ is an octagon. Identifying its opposite sides by translation as in Figure 2, we obtain a Riemann surface $X$ of genus two. The form $d z$ on $\mathbb{C}$ descends to a holomorphic 1 -form $\omega$ on $X$, with a double zero at the single point of $X$ coming from the vertices of $P$.

A pentagon is determined by five complex numbers satisfying $\sum v_{i}=0$. The parameters $\left(v_{i}\right)$ are just the periods of $\omega$ along the loops in $X$ coming from edges of $P$. By varying the shape of the pentagon $P$, we obtain a local holomorphic map of $\mathbb{C}^{4}$ into $\Omega \mathcal{T}_{2}$, providing a chart for $\Omega \mathcal{T}_{2}(2)$ near $(X, \omega)$.

The action of $A \in \mathrm{GL}_{2}^{+}(\mathbb{R})$ is transparent in this construction: if we start with the pentagon $A(P)$ instead of $P$, the resulting point in $\Omega \mathcal{T}_{2}$ is $A \cdot(X, \omega)$. 
Complex geodesics. Now consider the complex geodesic

$$
f: \mathbb{H} \rightarrow \mathcal{M}_{g}
$$

generated by $(X, q)$, where $q=\omega^{2}$ is the square of a holomorphic 1 -form. Then $f$ is simply the projection of the orbit of $(X, \omega)$ under $\mathrm{SL}_{2}(\mathbb{R})$; it is the unique map making the diagram

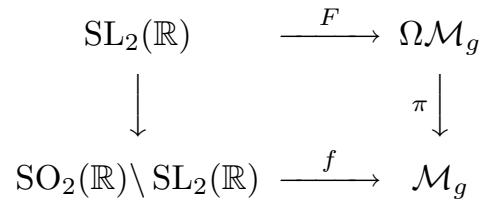

commute, where $F(A)=A \cdot(X, \omega)$.

In more concrete terms, for any $t \in \mathbb{H}$ define

$$
f(t)=\pi\left(A_{t} \cdot(X, \omega)\right)
$$

where $A_{t}: \mathbb{C} \rightarrow \mathbb{C}$ is the real-linear map given by

$$
A_{t}(x+i y)=x+t y=\left(\begin{array}{ll}
1 & \operatorname{Re} t \\
0 & \operatorname{Im} t
\end{array}\right)\left(\begin{array}{l}
x \\
y
\end{array}\right) .
$$

Then $X_{t}=f(t)$ is the Riemann surface obtained by pulling the complex structure on $\mathbb{C}$ back to $\widetilde{X}$ via the map

$$
\widetilde{X} \stackrel{I_{\omega}}{\longrightarrow} \mathbb{C} \stackrel{A_{t}}{\longrightarrow} \mathbb{C}
$$

and letting it descend to a new complex structure on $X$. The complex dilatation of the natural affine map $\phi_{t}: X \rightarrow X_{t}$ is given by

$$
\mu\left(\phi_{t}\right)=\frac{d A_{t} / d \bar{z}}{d A_{t} / d z} \cdot \frac{\bar{\omega}}{\omega}=\left(\frac{i-t}{i+t}\right) \cdot \frac{\bar{\omega}}{\omega},
$$

in accordance with (2.1).

Affine automorphisms of $(X, \omega)$. For 1-forms, we can also describe $\operatorname{Stab}(f)$ directly in terms of the affine geometry of $(X, \omega)$.

Let $\operatorname{Aff}(X, \omega)$ denote the group of affine automorphisms $\phi:(X, \omega) \rightarrow(X, \omega)$ and $\operatorname{GL}(X, \omega)$ its image under $\phi \mapsto D \phi$. Since $\int_{X}|\omega|^{2}$ is preserved, we have $\operatorname{det} D \phi=$ \pm 1 .

Let $\operatorname{SL}(X, \omega)$ denote the image of the orientation-preserving subgroup $\mathrm{Aff}^{+}(X, \omega)$ in $\mathrm{SL}_{2}(\mathbb{R})$. We have an exact sequence:

$$
0 \rightarrow \operatorname{Aut}(X, \omega) \rightarrow \operatorname{Aff}^{+}(X, \omega) \rightarrow \mathrm{SL}(X, \omega) \rightarrow 0,
$$

where $\operatorname{Aut}(X, \omega)$ is the finite group of conformal automorphisms of $X$ preserving $\omega$. The image of the inclusion

$$
\operatorname{Aff}^{+}(X, \omega) \subset \operatorname{Mod}\left(Z_{g}\right)
$$

is the stabilizer of $\widetilde{f}(\mathbb{H}) \subset \mathcal{T}_{g}$, while $\operatorname{Aut}(X, \omega)$ maps to the finite subgroup of $\operatorname{Mod}\left(Z_{g}\right)$ fixing $\widetilde{f}(\mathbb{H})$ pointwise.

Proposition 3.2. The complex geodesic generated by $\left(X, \omega^{2}\right)$ satisfies

$$
\operatorname{Stab}(f)=R \cdot \operatorname{SL}(X, \omega) \cdot R
$$

in $\mathrm{PSL}_{2}(\mathbb{R})$, where $R \in \mathrm{GL}_{2}(\mathbb{R})$ is the involution $\left(\begin{array}{cc}-1 & 0 \\ 0 & 1\end{array}\right)$. 
Proof. The map $f: \mathbb{H} \rightarrow \mathcal{M}_{g}$ has a natural lift

$$
\tilde{f}: \mathbb{H} \rightarrow \mathbb{P} \Omega \mathcal{M}_{g}=\left(\mathbb{R}^{*} \cdot \mathrm{SO}_{2}(\mathbb{R})\right) \backslash \Omega \mathcal{M}_{g}
$$

with the same stabilizer, given by $\widetilde{f}(t)=\left[A_{t} \cdot(X, \omega)\right]$. This map satisfies $\widetilde{f}(t)=\widetilde{f}(s)$ iff there is a $B \in \mathrm{SL}(X, \omega)$ such that $\left[A_{t} B\right]=\left[A_{s}\right]$ in $\mathrm{SO}_{2}(\mathbb{R}) \backslash \mathrm{SL}_{2}(\mathbb{R})$. By a direct computation (using the fact that $A_{t}^{-1}(i)=-\bar{t}$ ), we find that $\left[A_{t} B\right]=\left[A_{s}\right]$ iff $t=(R B R)(s)$, which gives (3.6).

Corollary 3.3. $\left(X, \omega^{2}\right)$ generates a Teichmüller curve iff $\operatorname{SL}(X, \omega)$ is a lattice in $\mathrm{SL}_{2}(\mathbb{R})$.

Primitivity. A Teichmüller curve in $\mathcal{M}_{h}$ generated by $\left(Y, \eta^{2}\right)$ gives rise to Teichmüller curves in $\mathcal{M}_{g}$ for infinitely many $g>h$, as follows. Suppose $\operatorname{SL}(Y, \eta)$ is a lattice. Choose a finite $\operatorname{SL}(Y, \eta)$-invariant set $B \subset Y$ and a subgroup of finite index in $\pi_{1}(Y-B)$. This information determines a Riemann surface $X$ with a proper map $\pi: X \rightarrow Y$ branched over $B$. Let $\omega=\pi^{*} \eta$. Then a finite index subgroup of $\operatorname{Aff}(Y, \eta)$ lifts to $X$, so $\operatorname{SL}(X, \omega)$ is also a lattice, commensurable to $\operatorname{SL}(Y, \eta)$. In this case we say that the pair $(X, \omega)$ and $(Y, \eta)$, as well as the pair of Teichmüller curves they generate, are commensurable.

A Teichmüller curve in $\mathcal{M}_{g}$ is primitive if it is not commensurable to a Teichmüller curve in $\mathcal{M}_{h}, h<g$.

Example: Branched covers of tori. The simplest Teichmüller curves arise in genus 1 . Indeed, for any complex torus $E=\mathbb{C} / L$, we have

$$
\mathrm{SL}(E, d z)=\mathrm{SL}_{2}(\mathbb{Z})
$$

thus $\left(E, d z^{2}\right)$ generates a Teichmüller curve $V$ mapping bijectively to $\mathcal{M}_{1}$. We say $(X, \omega)$ arises via a torus if it is commensurable to $(E, d z)$ for some complex torus $E$, equivalently, if $\omega=\pi^{*}(d z)$ where $\pi: X \rightarrow E$ is branched over torsion points on E.

We will be mostly interested in primitive Teichmüller curves, so it is useful to be able to recognize those arising via tori. For example, such curves are characterized by the property that the trace field of $\operatorname{SL}(X, \omega)$ is $\mathbb{Q}$ (see $\$ 5$ ).

Note. The reflection in (3.6) arises from the following fact. Let $K=\mathrm{SO}_{2}(\mathbb{R}) \subset G=$ $\mathrm{SL}_{2}(\mathbb{R})$. There are two natural complex structures on $G / K$. One arises from the map $G / K \cong \mathbb{H}$ sending $g$ to $g(i)$. The other arises from the map $G / K \cong \Delta$ sending $g$ to $\mu\left(g^{-1}\right)$, the Beltrami coefficient of $g^{-1}: \mathbb{R}^{2} \rightarrow \mathbb{R}^{2}$. These two structures are different; the map $\mathbb{H} \rightarrow \Delta$ they determine is anti-holomorphic.

For more on spaces of holomorphic 1-forms and quadratic differentials, see for example [Mas1], $\mathrm{V2}$, $\mathrm{KO}, \mathrm{EO}$ and $[\mathrm{KZ}]$.

\section{Abelian varieties}

In this section we show the complex geodesic generated by a 1-form maps isometrically not just to $\mathcal{M}_{g}$ but to $\mathcal{A}_{g}$. 
Abelian varieties. Let $\mathfrak{H}_{g}$ denote the Siegel upper half-space of $g \times g$ complex matrices $\tau$ with $\operatorname{Im}(\tau)$ positive-definite. The quotient $\mathcal{A}_{g}=\mathfrak{H}_{g} / \mathrm{Sp}_{2 g}(\mathbb{Z})$ is the moduli space of principally polarized Abelian varieties.

There is a natural injective holomorphic map $\mathcal{M}_{g} \rightarrow \mathcal{A}_{g}$ sending $X$ to its Jacobian variety,

$$
\operatorname{Jac}(X)=\Omega(X)^{*} / H_{1}(X, \mathbb{Z}) .
$$

To describe this map concretely, choose a symplectic basis $\left(a_{i}, b_{i}\right)_{1}^{g}$ for $H_{1}\left(Z_{g}, \mathbb{Z}\right)$ (such that $a_{i} \cdot b_{i}=-b_{i} \cdot a_{i}=1$ and all other products vanish). Given $X \in \mathcal{T}_{g}$, let $\omega_{i}$ be a basis for $\Omega(X)$ normalized so that $\int_{a_{i}} \omega_{j}=\delta_{i j}$, using the marking to identify $H_{1}\left(Z_{g}, \mathbb{Z}\right)$ and $H_{1}(X, \mathbb{Z})$. Then the period matrix

$$
\tau_{i j}(X)=\int_{b_{j}} \omega_{i}
$$

lies in the Siegel upper half-space, and we have

$$
\operatorname{Jac}(X) \cong \mathbb{C}^{g} /\left(\mathbb{Z}^{g} \oplus \tau \mathbb{Z}^{g}\right) .
$$

The map $\mathcal{T}_{g} \rightarrow \mathfrak{H}_{g}$ given by $X \mapsto \tau(X)$ covers the natural inclusion $\mathcal{M}_{g} \rightarrow \mathcal{A}_{g}$.

Theorem 4.1. Let $\mathbb{H} \rightarrow \mathcal{M}_{g}$ be the complex geodesic generated by $\left(X, \omega^{2}\right), \omega \in$ $\Omega(X)$. Then the composed map

$$
\mathbb{H} \rightarrow \mathcal{M}_{g} \rightarrow \mathcal{A}_{g}
$$

is an isometry for the Kobayashi metrics on domain and range.

Proof. Let $\tilde{f}: \mathbb{H} \rightarrow \mathcal{T}_{g}$ denote a lift of the complex geodesic generated by $\left(X, \omega^{2}\right)$. Then

$$
\tau \circ \tilde{f}: \mathbb{H} \rightarrow \mathfrak{H}_{g}
$$

is a lift of the map $\mathbb{H} \rightarrow \mathcal{M}_{g} \rightarrow \mathcal{A}_{g}$, so it suffices to check that $\tau \circ \tilde{f}$ is an isometry.

Choose a symplectic basis $\left(a_{i}^{\prime}, b_{i}^{\prime}\right)$ for $H_{1}\left(Z_{g}, \mathbb{R}\right)$ such that $\int_{a_{i}^{\prime}} \omega=\delta_{i 1}$. Define a $\operatorname{map} \sigma: \mathcal{T}_{g} \rightarrow \mathfrak{H}$ by

$$
\sigma_{i j}(Y)=\int_{b_{i}^{\prime}} \omega_{j}
$$

where $\omega_{j} \in \Omega(Y)$ are normalized so that $\int_{a_{i}^{\prime}} \omega_{j}=\delta_{i j}$. Since $\left(a_{i}, b_{i}\right)$ and $\left(a_{i}^{\prime}, b_{i}^{\prime}\right)$ are related by a symplectic automorphism of $H_{1}\left(Z_{g}, \mathbb{R}\right)$, there is a matrix $T \in$ $\operatorname{Sp}_{2 g}(\mathbb{R})=\operatorname{Aut}\left(\mathfrak{H}_{g}\right)$ such that

$$
\sigma(Y)=T \cdot \tau(Y)
$$

In particular, $\sigma$ is holomorphic.

Let $\left(X_{t}, \omega_{t}\right)=A_{t} \cdot(X, \omega)$, so that $X_{t}=\widetilde{f}(t)$; and let $\sigma_{11}(X)=x+i y$. By equation (3.3) we have

$$
\sigma_{11}\left(X_{t}\right)=\frac{\int_{b_{1}^{\prime}} \omega_{t}}{\int_{a_{1}^{\prime}} \omega_{t}}=\frac{A_{t}(x+i y)}{A_{t}(1)}=x+t y .
$$

Let $\tau_{11}: \mathfrak{H}_{g} \rightarrow \mathbb{H}$ be the coordinate function giving the first diagonal entry of $\tau \in \mathfrak{H}_{g}$. It takes values in $\mathbb{H}$ because $\operatorname{Im}(\tau)$ is positive-definite. The composed mapping

$$
\mathbb{H} \stackrel{\tilde{f}}{\longrightarrow} \mathcal{T}_{g} \stackrel{\sigma}{\longrightarrow} \mathfrak{H}_{g} \stackrel{\tau_{11}}{\longrightarrow} \mathbb{H}
$$


is then an isometry from $\mathbb{H}$ to $\mathbb{H}$, since it is given by

$$
\tau_{11} \circ \sigma \circ \tilde{f}(t)=\sigma_{11}\left(X_{t}\right)=x+t y .
$$

Now a composition of holomorphic maps is a Kobayashi isometry if and only if each factor is. Thus $\sigma \circ \widetilde{f}: \mathbb{H} \rightarrow \mathfrak{H}_{g}$ is also a Kobayashi isometry. Since $\sigma \circ \widetilde{f}$ and $\tau \circ \widetilde{f}$ differ by an isometry of $\mathfrak{H}_{g}$, the latter map is an isometry as well.

Contraction. Although the map $\tau \circ f: \mathbb{H} \rightarrow \mathfrak{H}_{g}$ generated by $\left(X, \omega^{2}\right)$ is an isometry, the periods of differentials other than $\omega$ move at speed strictly less than one.

To make this precise, let $X_{t}=\widetilde{f}(t) \in \mathcal{T}_{g}$ be the complex geodesic generated by $\left(X, \omega^{2}\right)$. Let $\left(a_{i}^{\prime}, b_{i}^{\prime}\right)$ be a symplectic basis for $H_{1}\left(Z_{g}, \mathbb{R}\right)$, and as in (4.1) define $\sigma_{i j}(Y)=\int_{b_{j}^{\prime}} \omega_{i}$ where $\omega_{i} \in \Omega(Y)$ satisfy $\int_{a_{i}^{\prime}} \omega_{j}=\delta_{i j}$.

Theorem 4.2. The holomorphic map $F: \mathbb{H} \rightarrow \mathbb{H}$ given by

$$
F(t)=\sigma_{i i}\left(X_{t}\right)
$$

is an isometry if $\omega_{i} \in \Omega(X)$ is proportional to $\omega$ and a strict contraction otherwise.

The proof will use Ahlfors' variational formula [Ah, §5], Roy. Let $\nu=\nu(z) d \bar{z} / d z$ be an $L^{\infty}$ Beltrami differential on $X$, representing a tangent vector to $\mathcal{T}_{g}$ at $X$. We then have:

$$
d \sigma_{i j}(\nu)=-2 i \int_{X} \omega_{i} \omega_{j} \nu
$$

Here the integrand is a symmetric tensor locally of the form $\omega_{i}(z) \omega_{j}(z) \nu(z)|d z|^{2}$.

Proof of Theorem 4.2. By (3.5) we have

$$
\nu=\left.\frac{d X_{t}}{d t}\right|_{t=i}=\frac{i}{2} \frac{\bar{\omega}}{\omega},
$$

and thus by Ahlfors' formula,

$$
\left.\frac{d F}{d t}\right|_{t=i}=\int_{X} \omega_{i}^{2} \cdot \frac{\bar{\omega}}{\omega} .
$$

By Riemann's bilinear relations we also have $\operatorname{Im}\left(\sigma_{i i}(X)\right)=\int_{X}\left|\omega_{i}\right|^{2}$. Thus the norm of $D F$ at $t=i$, using the hyperbolic metric $|d z| / \operatorname{Im} z$ on the domain and range, is given by

$$
\|D F\|=\left|\int_{X} \omega_{i}^{2} \cdot \frac{\bar{\omega}}{\omega}\right| / \int_{X}\left|\omega_{i}\right|^{2} .
$$

Since $|\bar{\omega} / \omega|=1$, we clearly have $\|D F\| \leq 1$, with equality iff $\omega_{i}$ is proportional to $\omega$. By the Schwarz lemma, $F$ is an isometry if $\|D F\|=1$ and a strict contraction otherwise.

Note. Theorem 4.1 was proved earlier in [Kra]; I am grateful to H. Miyachi for this reference.

\section{TRACE FIELDS}

In this section we indicate some general properties of the trace field of $\operatorname{SL}(X, \omega)$. 
Algebraic properties. The trace field of a group $\Gamma \subset \mathrm{SL}_{2}(\mathbb{R})$ is the subfield of $\mathbb{R}$ generated by $\operatorname{tr}(A), A \in \Gamma$.

Fix $(X, \omega) \in \Omega \mathcal{M}_{g}$. The group $\operatorname{Aff}(X, \omega)$ acts by pullback on $H^{1}(X, \mathbb{R})$, preserving $H^{1}(X, \mathbb{Z})$ as well as the real subspace $S \subset H^{1}(X, \mathbb{R})$ spanned by $(\operatorname{Re} \omega, \operatorname{Im} \omega)$.

Let $K \subset \mathbb{R}$ be the algebra generated over $\mathbb{Q}$ by traces of elements of $\operatorname{SL}(X, \omega)$. Any $t \in K$ has the form of a finite sum,

$$
t=\sum a_{i} \operatorname{tr}\left(D \phi_{i}\right), \quad a_{i} \in \mathbb{Q}, \quad \phi_{i} \in \operatorname{Aff}(X, \omega) .
$$

Since $\operatorname{tr}(D \phi)=\operatorname{tr}\left(\phi^{*} \mid S\right)$, we see $K$ is a quotient of the $\mathbb{Q}$-algebra of endomorphisms of $H^{1}(X, \mathbb{Q})$ generated by $\operatorname{Aff}(X, \omega)$. The latter algebra is finite-dimensional over $\mathbb{Q}$, so $K$ is as well. Thus $K$ is a number field, coinciding with the trace field of $\mathrm{SL}(X, \omega)$.

Theorem 5.1. The trace field of $\operatorname{SL}(X, \omega)$ has degree at most $g$ over $\mathbb{Q}$, where $g$ is the genus of $X$.

Proof. By the theorem of the primitive element, $K=\mathbb{Q}(t)$ for some $t=\sum a_{i} \operatorname{tr}\left(D \phi_{i}\right)$. Consider the endomorphism of $H^{1}(X, \mathbb{Q})$ given by

$$
T=\sum a_{i}\left(\phi_{i}^{*}+\left(\phi_{i}^{-1}\right)^{*}\right) .
$$

Since $A+A^{-1}=(\operatorname{tr} A) I$ for $A \in \mathrm{SL}_{2}(\mathbb{R})$, we have $T \mid S=t I$. Thus $t$ is an eigenvalue of $T$ of multiplicity at least 2 . It follows that the square of the characteristic polynomial for $t$ divides the degree $2 g$ polynomial $\operatorname{det}(x I-T)$. Thus $\operatorname{deg}(K / \mathbb{Q})=$ $\operatorname{deg}(t / \mathbb{Q}) \leq g$.

Since the spectrum of $A=D \phi$ is contained in the spectrum of $\phi^{*} \mid H^{1}(X, \mathbb{Z})$, we have:

Theorem 5.2. The traces of elements of $\operatorname{SL}(X, \omega)$ are algebraic integers.

Spectral radius. We next observe that the eigenvalues of $A=D \phi$ are extremal among their Galois conjugates and among the eigenvalues of $\phi^{*}$.

Theorem 5.3. Suppose $A=D \phi \in \operatorname{SL}(X, \omega)$ is hyperbolic. Then:

(1) the map $\phi^{*} \mid H^{1}(X, \mathbb{R})$ has a simple eigenvalue $\lambda \in \mathbb{R}$ such that $|\lambda|>\left|\lambda^{\prime}\right|$ for all other eigenvalues $\lambda^{\prime}$; and

(2) the eigenvalues of $A$ are $\lambda^{ \pm 1}$.

Proof. These results follow from basic results in the theory of pseudo-Anosov maps; cf. [FLP], Pen].

Let $\lambda^{ \pm 1} \in \mathbb{R}$ be the eigenvalues of $A$, where $|\lambda|>1$. Since $A$ is hyperbolic, $\phi$ is pseudo-Anosov. Since $\phi$ is real-affine with respect to $\omega$, its expanding and contracting measured foliations represent cohomology classes

$$
\mu_{ \pm} \in S \subset H^{1}(X, \mathbb{R})
$$

where $S$ is the span of $\operatorname{Re} \omega$ and $\operatorname{Im} \omega$. These classes are eigenvectors: they satisfy $\phi^{*} \mu_{ \pm}=\lambda^{ \pm 1} \mu_{ \pm}$.

Now consider any cohomology class $C \neq 0$ in $H^{1}(X, \mathbb{Z})$, Poincaré dual to a simple closed curve. Under iteration, this curve is stretched along the expanding foliation of $\phi$ and contracted in other directions, so as $n \rightarrow \infty$, we have $\lambda^{-n}\left(\phi^{n}\right)^{*} C \rightarrow t \mu_{+}$ for some $t \neq 0$. Thus $t \mu_{+}$is the only eigencomponent of $C$ with an eigenvalue satisfying $\left|\lambda^{\prime}\right| \geq|\lambda|$. Since the classes $C$ dual to simple closed curves span $H^{1}(X, \mathbb{Z})$, we conclude that $\lambda$ is a simple eigenvalue dominating all others. 
A Perron number is an algebraic integer $\lambda \geq 1$ whose other conjugates satisfy $\left|\lambda^{\prime}\right|<\lambda$. These are exactly the numbers that arise as the leading eigenvalues of Perron-Frobenius matrices [Li].

Theorem 5.4. The spectral radius of any $A=D \phi \in \mathrm{SL}(X, \omega)$ satisfies

$$
\rho(A)=\rho\left(\phi^{*} \mid H^{1}(X, \mathbb{Z})\right),
$$

and $\rho(A)^{2}$ is a Perron number.

Proof. When $A$ is hyperbolic, this theorem is immediate from the preceding result, using the fact that the spectrum of $\phi^{*} \mid H^{1}(X, \mathbb{Z})$ is closed under Galois conjugation and contains the eigenvalues of $A$. The elliptic and parabolic cases are also straightforward: in these cases, $\rho(A)=1$.

Recognizing branched covers. Finally we note that branched covers of tori can be detected via the trace field.

Theorem 5.5 (Gutkin-Judge). A lattice $\mathrm{SL}(X, \omega)$ arises via a torus if and only if its trace field is $\mathbb{Q}$.

This result follows from Theorems 5.5 and 7.1 of [GJ].

\section{Hilbert modular varieties}

In this section we discuss the connection between Hilbert modular varieties and real multiplication, summarized by:

Theorem 6.1. The set of principally polarized Abelian varieties admitting real multiplication is parameterized by a countable set of Hilbert modular varieties $\Sigma \rightarrow$ $\mathcal{A}_{g}$.

These Hilbert modular varieties can be considered as higher-dimensional analogues of Teichmüller curves.

We also examine curves whose Jacobians admit real multiplication and show their eigenforms are always primitive.

Real multiplication. Let $K$ be a totally real number field of degree $g$ over $\mathbb{Q}$.

Let $A=\mathbb{C}^{g} / L$ be a complex torus, and let $\operatorname{End}(A)$ denote its ring of endomorphisms. We regard $T \in \operatorname{End}(A)$ as a homomorphism $T: L \rightarrow L$ that extends to a complex-linear map under the embedding $L \hookrightarrow \mathbb{C}^{g}$. Similarly $\operatorname{End}(A) \otimes \mathbb{Q}$ consists of complex-linear endomorphisms of $L \otimes \mathbb{Q}$.

A polarization for $A$ is a choice of a symplectic form $E: L \times L \rightarrow \mathbb{Z}$, compatible with the complex structure. Extending $E$ to $L \otimes \mathbb{R} \cong \mathbb{C}^{g}$, this compatibility means $E(z, w)=E(i z, i w)$ and $E(z, i z)>0$ if $z \neq 0$; equivalently, $E$ gives a Kähler metric on $A$.

A polarized Abelian variety $A$ admits real multiplication by $K$ if there is an inclusion of $\mathbb{Q}$-algebras

$$
K \rightarrow \operatorname{End}(A) \otimes \mathbb{Q}
$$

such that

$$
E(k x, y)=E(x, k y)
$$

for all $k \in K$ and $x, y \in L \otimes \mathbb{Q}$. Equivalently, $\operatorname{End}(A)$ contains a copy of an order $\mathfrak{o} \subset K$ acting by self-adjoint endomorphisms. Compare [Rap, Def. 1.8]. 
From a dynamical perspective, the elements of $\mathfrak{o}$ (other than $0, \pm 1$ ) act on $A$ by Anosov endomorphisms, preserving a system of $g$ orthogonal 1-dimensional complex foliations. These foliations come from the eigenspaces $S_{i}$ of $K$ we will consider below.

Note that if $S$ is a symplectic vector space with $\operatorname{dim}_{\mathbb{R}} S=2$, then the only self-adjoint endomorphisms of $S$ are multiples of the identity.

Hilbert modular varieties. Using the $g$ embeddings of $K$ into $\mathbb{R}$, we can regard $\mathrm{SL}_{2}(K)$ as a subgroup of $\mathrm{SL}_{2}(\mathbb{R})^{g} \subset \operatorname{Aut}\left(\mathbb{H}^{g}\right)$.

A Hilbert modular variety $\Sigma=\mathbb{H}^{g} / \Gamma$ is a quotient of $\mathbb{H}^{g}$ by a lattice $\Gamma \subset \mathrm{SL}_{2}(K)$. A typical example is provided by $\Gamma=\mathrm{SL}_{2}\left(\mathcal{O}_{K}\right)$, where $\mathcal{O}_{K}$ is the ring of integers in $K$.

These varieties arise naturally in connection with real multiplication, as follows. Let $E_{0}$ denote the standard symplectic form on $L_{0}=\mathbb{Z}^{2 g}$, satisfying $E_{0}\left(a_{i}, b_{j}\right)=\delta_{i j}$. Choose an embedding

$$
\iota: K \rightarrow \operatorname{End}\left(L_{0}\right) \otimes \mathbb{Q}
$$

such that $E_{0}(k x, y)=E_{0}(x, k y)$ for all $k \in K$. Because of this self-adjointness, we can find a $K$-linear isomorphism

$$
L_{0} \otimes \mathbb{Q} \cong K^{2}
$$

sending $E_{0}$ to the standard symplectic form $E_{1}$ on $K^{2}$, given by

$$
E_{1}(k, \ell)=\operatorname{Tr}_{\mathbb{Q}}^{K} \operatorname{det}\left(\begin{array}{ll}
k_{1} & k_{2} \\
\ell_{1} & \ell_{2}
\end{array}\right) .
$$

Since $\mathrm{SL}_{2}(K)$ stabilizes the form above, we obtain an inclusion

$$
j: \mathrm{SL}_{2}(K) \rightarrow \mathrm{Sp}\left(L_{0} \otimes \mathbb{Q}\right) \cong \mathrm{Sp}_{2 g}(\mathbb{Q}) .
$$

Let $\Gamma \subset \mathrm{SL}_{2}(K)$ be the preimage of $\operatorname{Sp}_{2 g}(\mathbb{Z})$ under $j$. It can be shown that $\Gamma$ is commensurable to $\mathrm{SL}_{2}\left(\mathcal{O}_{K}\right)$; in particular, it is a lattice. Let $\Sigma=\mathbb{H}^{g} / \Gamma$ be the corresponding Hilbert modular variety.

Maps to $\mathcal{A}_{g}$. We now construct a natural map $f: \Sigma \rightarrow \mathcal{A}_{g}$ parameterizing certain Abelian varieties with real multiplication by $K$.

Let $\left(k_{1}, \ldots, k_{g}\right) \in \mathbb{R}^{g}$ denote the image of $k \in K$ under the $g$ field embeddings $K \hookrightarrow \mathbb{R}$. The isomorphism (6.2) between $L_{0} \otimes \mathbb{Q}$ and $K^{2}$ determines a splitting

$$
L_{0} \otimes \mathbb{R} \cong K^{2} \otimes_{\mathbb{Q}} \mathbb{R} \cong \bigoplus_{1}^{g} S_{i}
$$

such that $k \cdot\left(s_{1}, \ldots, s_{g}\right)=\left(k_{1} s_{1}, \ldots, k_{g} s_{g}\right)$. Since the action of $K$ is self-adjoint, the subspaces $S_{i} \cong \mathbb{R}^{2}$ are orthogonal with respect to the symplectic form $E_{0}$. If we now choose isomorphisms $S_{i} \cong \mathbb{C}$, preserving orientation, we obtain an embedding

$$
L_{0} \subset \bigoplus_{1}^{g} S_{i} \cong \mathbb{C}^{g}
$$

such that $K$ acts complex-linearly; thus $A=\mathbb{C}^{g} / L_{0}$ admits real multiplication by $K$. 
To connect this construction with the Siegel space, define $\delta: \mathbb{H}^{g} \rightarrow \mathfrak{H}_{g}$ by sending $t$ to the diagonal matrix

$$
\delta(t)=\delta\left(t_{1}, \ldots, t_{g}\right)=\left(\begin{array}{ccc}
t_{1} & & 0 \\
& \ddots & \\
0 & & t_{g}
\end{array}\right) \in \mathfrak{H}_{g} .
$$

The basis $a=(1,0), b=(0,1)$ for $K^{2}$ (satisfying $\left.E_{1}(a, b)=g\right)$ determines a symplectic basis $\left(a_{i}^{\prime}, b_{i}^{\prime}\right)$ for each $S_{i}$ (satisfying $E_{1}\left(a_{i}^{\prime}, b_{i}^{\prime}\right)=1$ ), and hence a symplectic basis for $L_{0} \otimes \mathbb{R}$. Let $T \in \operatorname{Sp}\left(L_{0} \otimes \mathbb{R}\right) \cong \mathrm{Sp}_{2 g}(\mathbb{R})$ be the unique transformation sending $\left(a_{i}^{\prime}, b_{i}^{\prime}\right)$ to the standard integral basis $\left(a_{i}, b_{i}\right)$, and define

$$
\tilde{f}: \mathbb{H}^{g} \rightarrow \mathfrak{H}_{g}
$$

by $\tilde{f}(t)=T \cdot \delta(t)$.

Proposition 6.2. For any $t \in \mathbb{H}^{g}$, the Abelian variety

$$
A=\mathbb{C}^{g} /\left(\mathbb{Z}^{g} \oplus \tau \mathbb{Z}^{g}\right),
$$

$\tau=\tilde{f}(t)$, admits real multiplication by $K$.

Proof. Since $\tau$ becomes $\delta(t)$ when expressed with respect to the basis $\left(a_{i}^{\prime}, b_{i}^{\prime}\right)$, it determines the unique complex structure on $\mathbb{R}^{2 g}=L_{0} \otimes \mathbb{R}$ such that $b_{i}^{\prime}=t_{i} a_{i}^{\prime}$. With respect to this structure, each subspace $S_{i}$ of $L_{0} \otimes \mathbb{R}$ is a complex line. Since $K$ acts on $S_{i}$ by a real scalar, its action on $L_{0} \otimes \mathbb{R}$ is complex-linear. Thus the map $\iota: K \rightarrow \operatorname{End}\left(L_{0}\right) \otimes \mathbb{Q}$ sends $K$ into $\operatorname{End}(A) \otimes \mathbb{Q}$, giving an action of $K$ by real multiplication on $A$.

Taking the quotient of the target of $\widetilde{f}$ by $\operatorname{Sp}_{2 g}(\mathbb{Z})$, we obtain a map

$$
f: \mathbb{H}^{g} \rightarrow \mathcal{A}_{g}
$$

satisfying $f(\gamma(t))=f(t)$ for all $\gamma \in \Gamma$. Indeed, we have $\tilde{f}(\gamma(t))=j(\gamma) \cdot \tilde{f}(t)$ for $\gamma \in \mathrm{SL}_{2}(K)$ by $(\underline{6.3})$, and $j(\gamma) \in \mathrm{Sp}_{2 g}(\mathbb{Z})$ if $\gamma \in \Gamma$. Thus $f$ descends to a map

$$
f: \Sigma \rightarrow \mathcal{A}_{g}
$$

on the Hilbert modular variety $\Sigma=\mathbb{H}^{g} / \Gamma$.

Like a Teichmüller curve, $f$ is an isometry for the Kobayashi metrics on its domain and range 11 Since $\Gamma$ is a lattice, it has finite index in $\operatorname{Stab}(f)$ and thus $f$ is finite-to-one.

Proof of Theorem 6.1. Let $A=\mathbb{C}^{g} / L$ be a principally polarized Abelian variety with real multiplication by $K$. Choose a symplectic isomorphism between $L$ and $L_{0}$. Then we obtain an inclusion

$$
\iota: K \rightarrow \operatorname{End}\left(L_{0}\right) \otimes \mathbb{Q} \cong \mathrm{M}_{2 g}(\mathbb{Q})
$$

as in (6.1). Since the eigenspaces of $K$ are complex-linear subspaces of $\mathbb{C}^{g} \cong L \otimes \mathbb{R}$, we have $[A] \in f(\Sigma)$ for the map $f: \Sigma \rightarrow \mathcal{A}_{g}$ constructed above.

There are only countably many fields $K$ of degree $g$ over $\mathbb{Q}$ and only countably many possibilities for $\iota: K \rightarrow \mathrm{M}_{2 g}(\mathbb{Q})$ given $K$. Hence the set of Abelian varieties with real multiplication by $K$ is parameterized by a countable collection of Hilbert modular surfaces.

\footnotetext{
${ }^{1}$ See Roy for the Kobayashi metric on $\mathfrak{H}_{g}$.
} 
For more details, see [Rap], $[\mathrm{HG}], \mathrm{vG}]$.

Eigenforms. Now let $\operatorname{Jac}(X)=\Omega(X)^{*} / H_{1}(X, \mathbb{Z})$ be the Jacobian of a curve with real multiplication by $K$. From the complex-linear action of $K$ on $\Omega(X)^{*}$ we obtain a dual action on $\Omega(X)$. A holomorphic 1-form $\omega \neq 0$ satisfying $K \cdot \omega \subset \mathbb{C} \cdot \omega$ is an eigenform for real multiplication by $K$.

We define the eigenform locus by

$$
\begin{aligned}
\mathcal{E}_{g}= & \left\{(X, \omega) \in \Omega \mathcal{M}_{g}: \operatorname{Jac}(X)\right. \text { admits real multiplication } \\
& \text { with } \omega \text { as an eigenform }\} .
\end{aligned}
$$

Theorem 6.3. Let $\omega$ be an eigenform for real multiplication by $K$ on $\operatorname{Jac}(X)$. Then $\omega$ does not arise as the pullback of a holomorphic 1-form from a Riemann surface of lower genus.

In particular, $\omega$ is primitive (in the sense of $₫ 3$ ).

Proof. Let $X$ have genus $g$. Choose an endomorphism $T: H^{1}(X, \mathbb{Q}) \rightarrow H^{1}(X, \mathbb{Q})$ generating the action of $K$ over $\mathbb{Q}$. Then the characteristic polynomial $P(x)=$ $\operatorname{det}(x I-T) \in \mathbb{Q}[x]$ is the square of an irreducible polynomial of degree $g$.

Now suppose $\omega$ is the pullback of a form on a surface $Y$ of genus $h$, under a map $\pi: X \rightarrow Y$. We will show $g=h$.

Via $\pi^{*}$ we can regard $H^{1}(Y, \mathbb{Q})$ as a subspace of $H^{1}(X, \mathbb{Q})$. Let

$$
S=\bigcap_{-\infty}^{\infty} T^{n}\left(H^{1}(Y, \mathbb{Q})\right)
$$

be the largest $T$-invariant subspace contained in $H^{1}(Y, \mathbb{Q})$. Since the eigenvectors $\omega$ and $\bar{\omega}$ for $T$ are contained in $H^{1}(Y, \mathbb{Q}) \otimes \mathbb{C}$, we have $2 \leq \operatorname{dim} S \leq 2 h$.

Because $S$ is $T$-invariant, $Q(x)=\operatorname{det}(x I-T \mid S)$ divides $P(x)$. Since $H^{1}(Y, \mathbb{Q})$ is invariant under complex conjugation, the eigenvalues of $T \mid S$ all have multiplicity two; thus $Q(X)=R(X)^{2}$, where $1 \leq \operatorname{deg} R \leq h$. But $P$ is irreducible, so we have $\operatorname{deg} R=g=h$.

\section{Curves of genus two}

Affine automorphisms of $X$ and real multiplication on $\operatorname{Jac}(X)$ are closely related when $X$ has genus two. In this section we will show:

Theorem 7.1. Let $X$ have genus two and let $K$ be the trace field of $\operatorname{SL}(X, \omega)$. If $K \neq \mathbb{Q}$, then $\operatorname{Jac}(X)$ admits real multiplication by $K$ with $\omega$ as an eigenform.

Theorem 7.2. The eigenform locus $\mathcal{E}_{2} \subset \Omega \mathcal{M}_{2}$ is $\mathrm{SL}_{2}(\mathbb{R})$-invariant.

In fact $\mathcal{E}_{2}$ is a countable union of 3-dimensional invariant submanifolds, lying over a countable union of Hilbert modular surfaces in $\mathcal{A}_{2}$.

Corollary 7.3. Let $(X, \omega)$ generate a primitive Teichmüller curve

$$
f: V \rightarrow \mathcal{M}_{2} \text {. }
$$


Then we have $(X, \omega) \in \mathcal{E}_{2}$, and the projection of $V$ to $\mathcal{A}_{2}$ factors through a Hilbert modular surface $\Sigma$, yielding the commutative diagram:

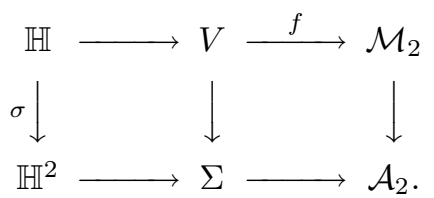

At the end of the section we show that these results break down in genus three; for example, $\mathcal{E}_{3}$ is not $\mathrm{SL}_{2}(\mathbb{R})$-invariant.

Genus two. The proofs of the theorems above use genus two in the following way.

Lemma 7.4. Let $A=\mathbb{C}^{2} / L$ be a polarized Abelian variety, and let $T: L \rightarrow L$ be a group homomorphism, self-adjoint with respect to the symplectic form. If $T$ leaves invariant a complex line

$$
\mathbb{C} \cong S \subset L \otimes \mathbb{R} \cong \mathbb{C}^{2}
$$

then we have $T \in \operatorname{End}(A)$.

Proof. Since $T$ is self-adjoint, it leaves invariant the splitting $\mathbb{C}^{2}=S \oplus S^{\perp}$ and acts by a real scalar on each factor. Since the symplectic form is compatible with the complex structure, $S^{\perp}$ is also a complex line. Thus $T$ is $\mathbb{C}$-linear, and therefore we have $T \in \operatorname{End}(A)$.

Proof of Theorem 7.1. Choose $A=D \phi \in \mathrm{SL}(X, \omega)$ such that $\operatorname{tr}(A)=t \notin \mathbb{Q}$. Let

$$
T=\phi_{*}+\phi_{*}^{-1}: H_{1}(X, \mathbb{Z}) \rightarrow H_{1}(X, \mathbb{Z}) .
$$

We claim $T$ is self-adjoint and $T \in \operatorname{End} \operatorname{Jac}(X)$.

To see that $T$ is self-adjoint, just note that $\phi_{*}$ preserves the intersection pairing $E$ on $H_{1}(X, \mathbb{Z})$. That is,

$$
E\left(\phi_{*} x, y\right)=E\left(x, \phi_{*}^{-1} y\right)
$$

and therefore $E(T x, y)=E(x, T y)$.

To see $T \in \operatorname{End}(A)$, recall that $\phi^{*}$ leaves invariant the subspace $S \subset H^{1}(X, \mathbb{R})$ spanned by $(\operatorname{Re} \omega, \operatorname{Im} \omega)$. Thus $\phi_{*}$ leaves invariant the subspace $\operatorname{Ann}(S) \subset H_{1}(X, \mathbb{R})$ of cycles $C$ such that

$$
\int_{C} \operatorname{Re} \omega=\int_{C} \operatorname{Im} \omega=0 .
$$

Under the identification of $H_{1}(X, \mathbb{R})$ with $\Omega(X)^{*}, \operatorname{Ann}(S)$ is the complex line of linear functionals vanishing on $\omega$. Thus $T$ leaves a complex line invariant, and therefore we have $T \in \operatorname{End}(\operatorname{Jac}(X))$ by the lemma above.

Passing to the dual, we obtain a self-adjoint complex linear endomorphism $T^{*}$ of $\Omega(X)$, satisfying $T^{*}(\omega)=t \omega$. In fact, with respect to an orthogonal basis $(\omega, \eta)$ for $\Omega(X)$, we obtain $T^{*}=\left(\begin{array}{cc}t & 0 \\ 0 & t^{\prime}\end{array}\right)$ where $t^{\prime}$ is the Galois conjugate of $t$. Thus $T$ generates a subalgebra $K$ of $\operatorname{End}(\operatorname{Jac}(X)) \otimes \mathbb{Q}$ isomorphic to the real quadratic field $K=\mathbb{Q}[t]$, and hence $\operatorname{Jac}(X)$ admits real multiplication with $\omega$ as an eigenform.

Proof of Theorem 7.2. Let $(Y, \eta)=A \cdot(X, \omega)$ with $A \in \mathrm{SL}_{2}(\mathbb{R})$ and $(X, \omega) \in \mathcal{E}_{2}$. Choose $T: H_{1}(X, \mathbb{Z}) \rightarrow H_{1}(X, \mathbb{Z})$ representing an element of $\operatorname{End}(\operatorname{Jac}(X))$ with eigenform $\omega$, such that $K=\mathbb{Q}[T]$ is a real quadratic field.

Let $S_{\omega}$ be the span of $(\operatorname{Re} \omega, \operatorname{Im} \omega)$ in $H^{1}(X, \mathbb{R})$, and similarly for $S_{\eta}$. We have a natural affine map $\phi:(X, \omega) \rightarrow(Y, \eta)$ sending $S_{\omega}$ to $S_{\eta}$. 
Since $\omega$ is an eigenform, $T$ leaves invariant the subspace $\operatorname{Ann}\left(S_{\omega}\right) \subset H_{1}(X, \mathbb{R})$ of cycles pairing trivially with $\omega$. Consequently the self-adjoint transformation

$$
U=\phi_{*} \circ T \circ \phi_{*}^{-1}
$$

of $H_{1}(Y, \mathbb{Z})$ leaves invariant the complex line $\operatorname{Ann}\left(S_{\eta}\right)$ in $H_{1}(Y, \mathbb{R}) \cong \Omega(Y)^{*}$. Thus we have $U \in \operatorname{End}(\operatorname{Jac}(Y))$ by the lemma above; and clearly $\eta$ is an eigenform of $U$. Since $\mathbb{Q}[T] \cong \mathbb{Q}[U]$, we have $(Y, \eta) \in \mathcal{E}_{2}$.

Proof of Corollary 7.3 Since $(X, \omega)$ is primitive, it does not arise via a torus, and thus the trace field $K$ of $\mathrm{SL}(X, \omega)$ is real quadratic by Theorem 5.5. By the preceding results, the projection of $V$ to $\mathcal{A}_{2}$ lies in the locus of Abelian varieties with real multiplication by $K$. The corollary then follows by the discussion of $₫ 6$ observing that a point in $V$ gives a Riemann surface $X$ together with a distinguished eigenform.

Instability of real multiplication. We conclude this section by showing that the results above are special to genus two.

Theorem 7.5. The eigenform locus $\mathcal{E}_{g}$ is not $\mathrm{SL}_{2}(\mathbb{R})$-invariant for $g=3$.

Proof. Let $\zeta=\exp (2 \pi i / 7)$ be a primitive 7 th root of unity. Consider the hyperelliptic curve $X$ of genus $g=3$ defined in affine coordinates by

$$
y^{2}=x^{7}-1
$$

Because of the automorphism $x \mapsto \zeta x, \operatorname{Jac}(X)$ admits complex multiplication by $\mathbb{Q}(\zeta)$. It therefore admits real multiplication by $K=\mathbb{Q}\left(\zeta+\zeta^{-1}\right)$. A basis of holomorphic eigenforms is given by

$$
\omega_{i}=x^{i-1} d x / y,
$$

$i=1,2,3$. Note that $\omega_{1} \omega_{3}=\omega_{2}^{2}$. In a symplectic basis adapted to the action of $K$, the period matrix $\sigma_{i j}(X)$ is diagonal.

Now consider the map $f: \mathbb{H} \rightarrow \Omega \mathcal{M}_{3}$ given by $f(t)=\left(X_{t}, \omega_{t}\right)=A_{t} \cdot\left(X, \omega_{2}\right)$. By Ahlfors' variational formula (4.2), we have

$$
\left.\frac{d \sigma_{13}\left(X_{t}\right)}{d t}\right|_{t=i}=\int_{X} \omega_{1} \omega_{3} \cdot \frac{\bar{\omega}_{2}}{\omega_{2}}=\int_{X} \omega_{2} \bar{\omega}_{2} \neq 0 .
$$

Thus for $t$ near $i \in \mathbb{H}, \sigma_{i j}\left(X_{t}\right)$ is no longer diagonal and $\left(X_{t}, \omega_{t}\right)$ is no longer an eigenform for the given action of $K$. This shows that some irreducible component of $\mathcal{E}_{3}$ fails to be $\mathrm{SL}_{2}(\mathbb{R})$-invariant. But if $\mathcal{E}_{3}$ as a whole is $\mathrm{SL}_{2}(\mathbb{R})$ invariant, the action must preserve components.

The same example shows that Theorem 7.1 also fails in genus three. The curve (7.1) above arises in a family of quotients of Fermat curves studied in GR]. For more on families of Jacobians with endomorphisms, see [CV], CVT].

\section{WEIERSTRASS FORMS}

A Weierstrass form is a holomorphic 1-form $\omega \in \Omega(X)$ whose zero divisor is concentrated at a single point. When $X$ has genus two, there are six such forms up to scale, one for each Weierstrass point. Let

$$
\begin{aligned}
\mathcal{W}_{2}= & \left\{X \in \mathcal{M}_{2}: \operatorname{Jac}(X)\right. \text { admits real multiplication with } \\
& \text { a Weierstrass eigenform }\}
\end{aligned}
$$


In this section we show:

Theorem 8.1. The locus $\mathcal{W}_{2} \subset \mathcal{M}_{2}$ is a countable union of primitive Teichmüller curves.

In the next section we will show that $\mathcal{W}_{2}$ has in fact infinitely many irreducible components (see Corollary 9.5).

Corollary 8.2. Let $K$ be the trace field of $\operatorname{SL}(X, \omega)$, where $\omega$ is a Weierstrass form and $X$ has genus two. If $K \neq \mathbb{Q}$, then $\operatorname{SL}(X, \omega)$ is a lattice.

Corollary 8.3. In genus two, a Weierstrass form generates a Teichmüller curve iff

- $(X, \omega)$ arises via a torus, or

- $(X, \omega)$ admits an affine automorphism with irrational trace.

We proceed to the proof of Theorem 8.1 .

Lemma 8.4. The locus $\mathcal{W}_{2} \subset \mathcal{M}_{2}$ is a countable union of algebraic subvarieties of $\mathcal{M}_{2}$.

Proof. We will cover $\mathcal{W}_{2}$ by the projections of countably many subvarieties of the algebraic $\mathbb{P}^{1}$-bundle $\mathbb{P} \Omega \mathcal{M}_{2}$ over $\mathcal{M}_{2}$.

Fix a real quadratic field $K$ and a self-adjoint embedding

$$
\iota: K \rightarrow \operatorname{End}\left(H_{1}\left(Z_{2}, \mathbb{Z}\right)\right) \cong \mathrm{M}_{4}(\mathbb{Q}) .
$$

Let $f: \Sigma_{\iota} \rightarrow \mathcal{A}_{2}$ be the Hilbert modular surface constructed in $\$ \underline{6}$, parameterizing Abelian varieties with real multiplication by $K$ via $\iota$. Then the map $f$ is finite and proper, and $f\left(\Sigma_{\iota}\right) \subset \mathcal{A}_{2}$ is an algebraic subvariety. Indeed, $f$ extends compatibly to compactifications of $\Sigma_{\iota}$ and $\mathcal{A}_{2}$, and $f\left(\Sigma_{\iota}\right)$ is a component of a Humbert surface; see [vG, $\S \mathrm{IX}]$, [HG, Ch. 7-9].

The natural map $\pi: \mathcal{M}_{g} \rightarrow \mathcal{A}_{g}$ is algebraic, so $R_{\iota}=\pi^{-1}\left(f\left(\Sigma_{\iota}\right)\right)$ is an algebraic subvariety of $\mathcal{M}_{2}$. Let $\mathcal{E}_{2}^{\iota} \subset \mathcal{E}_{2}$ be the set of pairs $(X, \omega)$ such that $\operatorname{Jac}(X) \in$ $f\left(\Sigma_{\iota}\right)$ and $\omega$ is an eigenvector for real multiplication by $K$ compatible with $\iota$. Projectivizing, we obtain a 2 -sheeted covering space of $R_{\iota}$, making the diagram

commute.

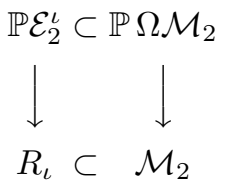

Similarly, $\mathbb{P} \Omega \mathcal{M}_{2}(2) \rightarrow \mathcal{M}_{2}$ is a 6 -sheeted covering space, whose fibers are the projective equivalence classes of Weierstrass forms. The algebraic structure on this bundle can be made explicit, using the fact that curves of genus two are hyperelliptic: namely the fiber over the curve $y^{2}=\prod_{1}^{6}\left(x-a_{i}\right)$ consists of the forms $\omega_{i}=\left(x-a_{i}\right) d x / y, i=1, \ldots, 6$.

The locus of pairs $(X,[\omega]) \in \mathbb{P} \Omega \mathcal{M}_{2}$ such that $\omega$ is a Weierstrass eigenform for the given action of $K$ is simply the intersection

$$
\mathcal{P}_{\iota}=\mathbb{P} \mathcal{E}_{2}^{\iota} \cap \mathbb{P} \Omega \mathcal{M}_{2}(2) .
$$

Since the projection $\mathbb{P} \Omega \mathcal{M}_{2} \rightarrow \mathcal{M}_{2}$ is proper, the image $\mathcal{W}_{2}^{\iota}$ of $\mathcal{P}_{\iota}$ is a subvariety of $\mathcal{M}_{2}$. Taking the union of all possible actions of real quadratic fields, we find that $\mathcal{W}_{2}=\bigcup_{\iota} \mathcal{W}_{2}^{\iota}$ is a countable union of algebraic varieties. 
Lemma 8.5. The locus $\mathcal{W}_{2}$ contains no 2-dimensional subvariety.

Proof. Continuing with the notation above, we will show $\mathcal{P}_{\iota}$ is 1-dimensional. Choose $t \in K$ such that $K=\mathbb{Q}(t)$ and we have

$$
T=\iota(t) \in \operatorname{End}\left(H_{1}\left(Z_{2}, \mathbb{Z}\right)\right) .
$$

Then there is a symplectic splitting

$$
H_{1}\left(Z_{2}, \mathbb{R}\right)=S_{1} \oplus S_{2}
$$

such that $T \mid S_{i}=t_{i} I, i=1,2$. The eigenvalues $\left(t_{1}, t_{2}\right)$ of $T$ are the images of $t$ under the two embeddings $K \rightarrow \mathbb{R}$. Let $\left(a_{i}^{\prime}, b_{i}^{\prime}\right)$ be a symplectic basis for $S_{i}$.

By assumption $\omega$ is an eigenform. Let us number the eigenvalues so that $T^{*}(\omega)=$ $t_{1} \omega$. Since $t_{1} \neq t_{2}$, the form $\omega$ pairs trivially with cycles in $S_{2}$. On the other hand, by Theorem 3.1 the pair $(X, \omega)$ is locally determined by the periods of $\omega$. Thus the map

$$
F(X,[\omega])=\left[\int_{a_{1}^{\prime}} \omega: \int_{b_{1}^{\prime}} \omega\right]
$$

gives a local homeomorphism from $\mathcal{P}_{\iota}$ to $\mathbb{P}^{1}$. It follows that $\mathcal{P}_{\iota}$ and its projection $\mathcal{W}_{2}^{\iota}$ are at most 1-dimensional, so the same is true of $\mathcal{W}_{2}$.

Proof of Theorem 8.1. The locus $\mathcal{W}_{2}$ is the projection to $\mathcal{M}_{2}$ of the intersection $\mathcal{E}_{2} \cap \Omega \mathcal{M}_{2}(2)$ of the eigenform locus and the Weierstrass stratum. By Theorem 7.2 both $\mathcal{E}_{2}$ and $\Omega \mathcal{M}_{2}(2)$ are $\mathrm{SL}_{2}(\mathbb{R})$-invariant, so $\mathcal{W}_{2}$ is a union of complex geodesics.

The image of any such geodesic $f: \mathbb{H} \rightarrow \mathcal{W}_{2}$ must be contained in an single irreducible subvariety $V^{\prime}$ of $\mathcal{W}_{2}$. By the preceding lemma, $V^{\prime}$ is a curve. Thus $f$ factors through a map to the normalization $V$ of $V^{\prime}$, and hence $V^{\prime}$ is a Teichmüller curve. By Theorem $6.3 V^{\prime}$ is primitive, since it is generated by an eigenform. Since $\mathcal{W}_{2}$ has only countably many irreducible components, it is covered by countably many such curves.

Proof of Corollary 8.2. Let $\left(X_{t}, \omega_{t}\right)=A_{t} \cdot(X, \omega)$ so that $f(t)=\left[X_{t}\right]$ gives the complex geodesic

$$
f: \mathbb{H} \rightarrow \mathcal{M}_{2}
$$

generated by $\left(X, \omega^{2}\right)$. Clearly $\omega_{t}$ is a Weierstrass differential for all $t$, and it is also an eigenform by Theorem 7.2 Thus $f$ maps $\mathbb{H}$ into $\mathcal{W}_{2}$. Since $\mathcal{W}_{2}$ is a union of countably many algebraic curves, the map $f$ must cover one of them, and thus $\mathrm{SL}(X, \omega)$ is a lattice.

Proof of Corollary 8.3 Combine Corollary 8.2 with Theorem 5.5 .

Remark. Smillie has shown that a complex geodesic $f: \mathbb{H} \rightarrow \mathcal{M}_{g}$ is a Teichmüller curve iff $f(\mathbb{H})$ is a closed subset of moduli space. The proof, sketched in [V4, p. 226], uses Ratner's theorem.

Using this result, one can prove Theorem 8.1 without appealing to the algebraic structure of moduli space. Indeed, the arguments above show that any irreducible component $V$ of $\mathcal{W}_{2}$ is a closed complex geodesic, and hence a Teichmüller curve by Smillie's theorem. 


\section{Billiards}

In this section we recall the connection between billiard tables and Teichmüller curves. Using L-shaped tables, we then construct infinitely many Teichmüller curves in $\mathcal{M}_{2}$, none of which arise via tori. Finally we present an algorithm to find $\operatorname{SL}(X, \omega)$ explicitly in many of these examples.

Billiards. Let $P \subset \mathbb{R}^{2} \cong \mathbb{C}$ be a compact polygon whose interior angles are rational multiplies of $\pi$. One can associate to $P$ a point $(X, \omega) \in \Omega \mathcal{M}_{g}$, as follows.

Let $G \subset \mathrm{O}_{2}(\mathbb{R})$ be the finite group generated by the linear parts of reflections in the sides of $P$. Take $|G|$ disjoint copies of $P$, each rotated by an element of $G$. Glue each edge $E$ of $g P$ to the edge $r E$ of $r g P$ by a translation, where $r \in G$ is reflection through $E$. The result is a compact Riemann surface

$$
X=\left(\coprod_{G} g \cdot P\right) / \sim .
$$

The holomorphic 1-form $d z$ on $\mathbb{C}$ is translation-invariant, so it descends to a natural 1-form $\omega$ on $X$. The zeros of $\omega$, if any, come from the vertices of $P$.

By construction, billiard paths reflecting off the sides of $P$ unfold to form geodesics on $X$ for the flat metric $|\omega|$.

We define the trace field of $P$ to be the trace field of $\operatorname{SL}(X, \omega)$.

Lattice polygons. We say $P$ is a lattice polygon if $\mathrm{SL}(X, \omega)$ is a lattice in $\mathrm{SL}_{2}(\mathbb{R})$, equivalently, if $(X, \omega)$ generates a Teichmüller curve. In this case:

- every billiard path in $P$ that does not meet a vertex is either closed or uniformly distributed in $P$; and

- the number of side patterns of closed billiard paths of length $\leq L$ satisfies $N(L) \sim c L^{2}$ for some constant $c(P)>0$.

These results are restatements of Veech's Theorem (\$2). The side pattern of a closed billiard path is the cyclically ordered sequence of edges of $P$ that it hits, and one should restrict attention to paths hitting an even number of sides.

The small surface. When the polygon $P$ has symmetries, it is often useful to consider the small surface $\left(X^{\prime}, \omega^{\prime}\right)$, obtained by further identifying $g P$ and $h P$ if they are equal up to translation in $\mathbb{C}$. There is a natural covering map $(X, \omega) \rightarrow$ $\left(X^{\prime}, \omega^{\prime}\right)$.

The affine group $\mathrm{SL}\left(X^{\prime}, \omega^{\prime}\right)$ of the small surface is commensurable to that of the large surface, so either can be studied to test if $P$ is a lattice polygon.

The regular pentagon. It is known that regular polygons are lattice polygons V1]. To illustrate our methods, we give a new proof of:

Theorem 9.1. The regular pentagon is a lattice polygon.

Proof. Let $(X, \omega)$ be the small surface associated to a regular pentagon $P \subset \mathbb{R}^{2}$. Then $(X, \omega)$ is obtained by gluing together the opposite edges of $P$ and $-P$, as in Figure 2 of 93 . The vertices of $P$ descend to a single point on $X$, so $\omega$ is a Weierstrass form. (In fact $X$ is isomorphic to the curve $y^{2}=x^{5}-1$, and $\omega$ is a multiple of the form $d x / y$.)

By rotating $P$, we obtain an order 5 symmetry of $(X, \omega)$ with $\operatorname{tr} D \phi=2 \cos (2 \pi / 5)$. Thus the trace field of $\operatorname{SL}(X, \omega)$ is irrational, and therefore $\operatorname{SL}(X, \omega)$ is a lattice, by Corollary 8.2 . 


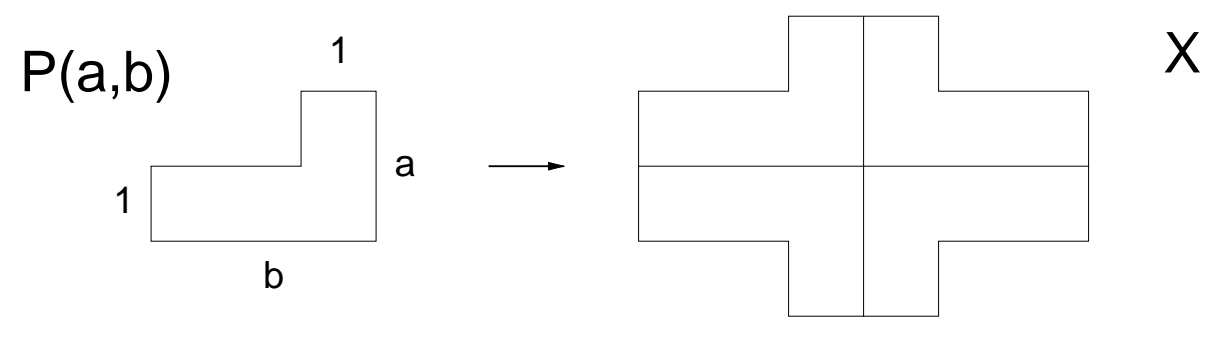

Figure 3. An L-shaped billiard table and the Riemann surface it determines.

L-shaped billiard tables. We now determine all the L-shaped lattice polygons.

An $L$-shaped polygon $P$ is obtained by removing a small rectangle from the corner of a larger rectangle. By applying a linear transformation $A \in \mathrm{GL}_{2}^{+}(\mathbb{R})$, any Lshaped polygon can be normalized so it takes the form $P(a, b)$ shown in Figure 3 with $a, b \geq 1$. The associated Riemann surface $X$ is obtained by forming a Swiss cross from four copies of $P(a, b)$ as shown at the right, then gluing opposite edges by horizontal and vertical translations. The natural 1-form $\omega$ on $X$ is a Weierstrass form; its unique zero comes from the vertex of $P$ with interior angle $3 \pi / 2$.

Theorem 9.2. The L-shaped polygon $P(a, b)$ is a lattice polygon iff $a$ and $b$ are rational or

$$
a=x+z \sqrt{d} \quad \text { and } \quad b=y+z \sqrt{d}
$$

for some $x, y, z \in \mathbb{Q}$ with $x+y=1$ and $d \geq 0$ in $\mathbb{Z}$. In the latter case the trace field of $P(a, b)$ is $\mathbb{Q}(\sqrt{d})$.

Corollary 9.3. $P(a, a)$ is a lattice polygon iff $a$ is rational or $a=(1 \pm \sqrt{d}) / 2$ for some $d \in \mathbb{Q}$.

Corollary 9.4. Every real quadratic field arises as the trace field of a Teichmüller curve in $\mathcal{M}_{2}$.

Corollary 9.5. There are infinitely many primitive Teichmüller curves in $\mathcal{M}_{2}$.

Since only finitely many real quadratic fields (those of the form $K=\mathbb{Q}(\cos (2 \pi / n)$ ), $n=5,8,10,12)$ arise as trace fields of triangle groups, we have:

Corollary 9.6. There exist Teichmüller curves $V=\mathbb{H} / \Gamma$ in $\mathcal{M}_{2}$ such that $\Gamma$ is not commensurable to a triangle group.

Lemma 9.7. Given $(X, \omega) \in \Omega \mathcal{M}_{g}$, suppose $X$ decomposes into a finite number of annuli $U_{i}$ foliated by the closed leaves of $\mathcal{F}\left(\omega^{2}\right)$. Then either:

- the moduli $m_{i}=\bmod \left(U_{i}\right)$ have rational ratios, and we have $A=\left(\begin{array}{ll}1 & 0 \\ t & 1\end{array}\right) \in$ $\mathrm{SL}(X, \omega)$ where $t=\operatorname{lcm}\left(m_{1}^{-1}, \ldots, m_{n}^{-1}\right)$; or

- the moduli $m_{i}$ have irrational ratios and $\mathrm{SL}(X, \omega)$ is not a lattice.

Proof. The first case follows by taking $A=D \phi$ where $\phi$ is a product of suitable Dehn twists in the annuli $U_{i}$.

For the second case, note that the real Teichmüller geodesic $f(i y)$ tends to infinity in $\mathcal{M}_{g}$ as $y \rightarrow \infty$; indeed, the hyperbolic lengths of the cores of the annuli $U_{i}$ tend to zero. If $V=\mathbb{H} / \mathrm{SL}(X, \omega)$ has finite volume, then [iy] must also exit a cusp of $V$. But then a horocycle around the cusp of $V$ gives rise to an affine automorphism $\phi$ 
of $(X, \omega)$ shearing along the leaves of $\mathcal{F}\left(\omega^{2}\right)$. A suitable power of $\phi$ effects Dehn twists on each $U_{i}$, so their moduli have rational ratios. Compare [V1, 2.4].

Proof of Theorem 9.2. We claim that $P(a, b)$ is a lattice polygon iff $a b-a$ and $a b-b$ are rational.

To see this, let $(X, \omega)$ be the Riemann surface associated to $P(a, b)$. Then $X$ has genus two and $\omega$ is a Weierstrass form.

The foliation $\mathcal{F}\left(\omega^{2}\right)$, whose leaves are vertical lines in the Swiss cross, decomposes $X$ into a pair of annuli with moduli $b-1$ and $1 / a$. By the lemma, for $\operatorname{SL}(X, \omega)$ to be a lattice we, must have $a b-a \in \mathbb{Q}$. Applying the same reasoning to the vertical foliation $\mathcal{F}\left(-\omega^{2}\right)$, we find $a b-b \in \mathbb{Q}$ as well.

For the converse, assume $a b-a$ and $a b-b$ are rational. Then $a-b$ is rational. If $a$ is rational, then so is $b$. In this case $P(a, b)$ can be tiled by squares, $(X, \omega)$ arises via a torus and $\mathrm{SL}(X, \omega)$ is a lattice commensurable to $\mathrm{SL}_{2}(\mathbb{Z})$.

Now assume $a b-a$ and $a b-b$ are rational but $a$ is irrational. By assumption, the reciprocal moduli $a$ and $1 /(b-1)$ of the vertical foliation have a rational ratio $a b-a$, and similarly for the horizontal foliation. Thus by the lemma we have

$$
\left(\begin{array}{cc}
1 & 0 \\
m a & 1
\end{array}\right),\left(\begin{array}{cc}
1 & n b \\
0 & 1
\end{array}\right) \in \operatorname{SL}(X, \omega)
$$

for some nonzero $m, n \in \mathbb{Z}$. Taking the trace of the product, we find that $2+$ mnab lies in the trace field $K$ of $\operatorname{SL}(X, \omega)$. But $a b-a$ is rational, so we have $a \in K$ and thus $K \neq \mathbb{Q}$. By Corollary 8.2, $\operatorname{SL}(X, \omega)$ is a lattice in this case as well.

To complete the proof, note that if $a$ and $b$ have the form given in the theorem, then $a b-a$ and $a b-b$ are rational. Conversely, if $a b-a$ and $a b-b$ are rational, then $a$ satisfies the quadratic equation

$$
a^{2}+a(b-a-1)+(a-a b)=0
$$

with rational coefficients. Thus $a=x+z \sqrt{d}$ for some $x, z \in \mathbb{Q}$ and $d \geq 0$ in $\mathbb{Z}$. Since $a-b$ is rational, we have $b=y+z \sqrt{d}$ for some $y \in \mathbb{Q}$. If $d$ is a square, then we have $a, b \in \mathbb{Q}$; otherwise the condition $a b-a \in \mathbb{Q}$ implies that $x+y=1$.

The golden table. We remark that the regular pentagon and the golden L-shaped table $P(\gamma, \gamma), \gamma=(1+\sqrt{5}) / 2$, generate the same Teichmüller curve $f: V \rightarrow \mathcal{M}_{2}$. In fact, as we will see below, $V$ is isomorphic to the $(2,5, \infty)$-orbifold. The map $f$ sends the singular points of orders 2 and 5 to the Riemann surfaces for $P(\gamma, \gamma)$ and for the regular pentagon, respectively. The relationship between the two polygons can be seen directly, as indicated in Figure 4.

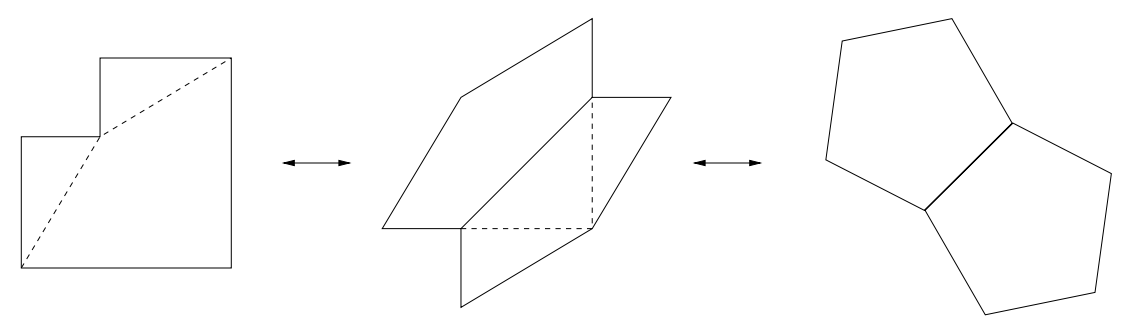

FiguRE 4. The golden L-shaped table can be reassembled and then stretched to obtain a pair of regular pentagons. 
Explicit uniformization. The proof that $\operatorname{SL}(X, \omega)$ is a lattice when its trace field is irrational is rather indirect. To conclude this section, we describe a direct algorithm to generate elements in $\operatorname{SL}(X, \omega)$. This algorithm allows one to verify:

Theorem 9.8. The Teichmüller curve generated by $P(a, a), a=(1+\sqrt{d}) / 2$, has genus 0 for $d=2,3,5,7,13,17,21,29$ and 33 .
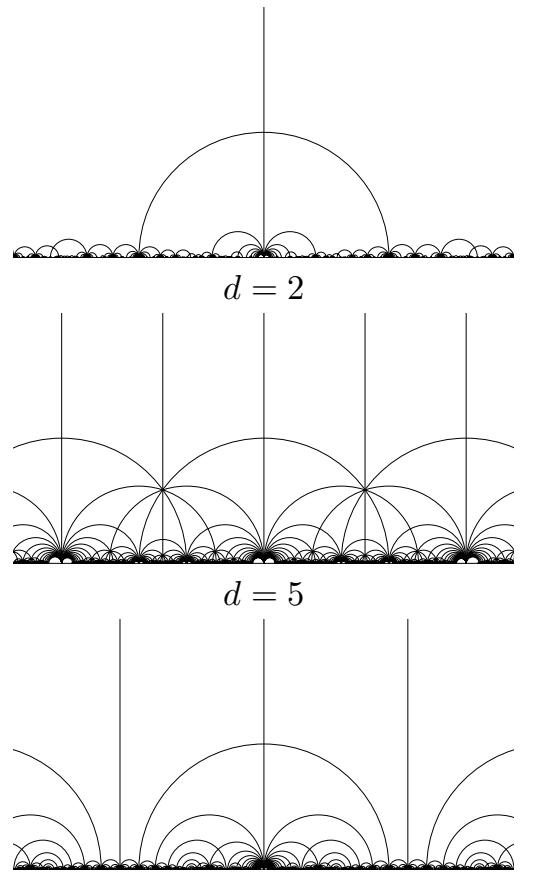

$d=13$
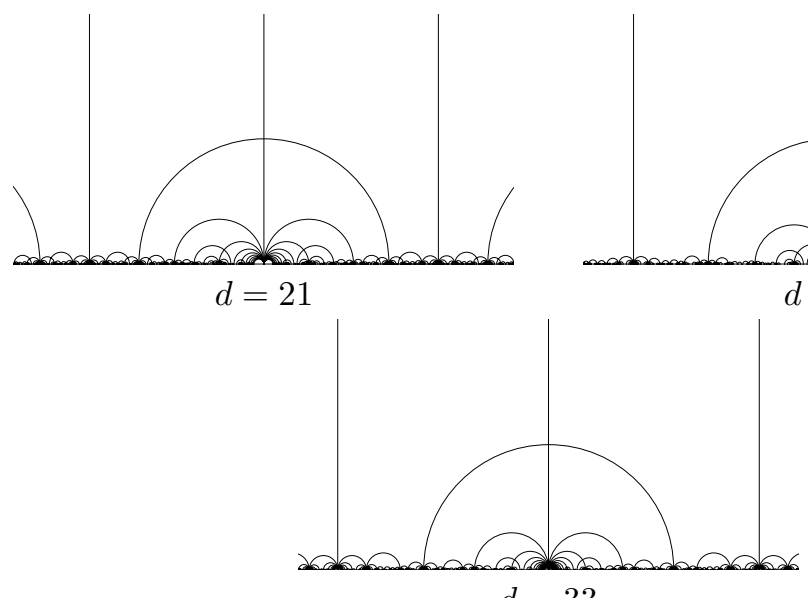

$d=33$

FiguRE 5. A sampling of Teichmüller curves.

To describe the algorithm, assume $(X, \omega)$ generates a Teichmüller curve $V=$ $\mathbb{H} / \operatorname{SL}(X, \omega)$. Let $\operatorname{GL}(X, \omega)$ denote the image of the full affine group $\operatorname{Aff}(X, \omega)$ 
under the map $\phi \mapsto D \phi$. Note that $\operatorname{GL}(X, \omega)$ contains $\operatorname{SL}(X, \omega)$ with index at most two.

To begin the algorithm, we must first choose an initial reflection $R_{0} \in \mathrm{GL}(X, \omega)$. Then the geodesic $\gamma_{0} \subset \mathbb{H}$ fixed by $R_{0}$ either covers a closed geodesic on $V$ or it joins a pair of cusps. Assume we are in the latter case, and let $c_{0}$ be one of the cusps fixed by $R_{0}$.

From the data $\left(R_{0}, c_{0}\right)$ we inductively generate a chain of cusps and reflections $\left(R_{i}, c_{i}\right)$, as follows.

(1) Choose a parabolic element $A_{i} \in \mathrm{SL}(X, \omega)$ stabilizing $c_{i}$.

(2) Set $R_{i+1}=A_{i} R_{i}$.

(3) Let $\gamma_{i+1} \subset \mathbb{H}$ be the axis of $R_{i+1}$.

(4) Let $c_{i+1}$ be the cusp joined to $c_{i}$ by $\gamma_{i+1}$.

Note that $R_{i+1}$ is indeed a reflection, since $A_{i} R_{i}=R_{i} A_{i}^{-1}$.

In terms of the affine surface $(X, \omega)$, each cusp $c_{i}$ determines an angle $\theta=$ $\tan ^{-1}\left(1 / c_{i}\right)$ such that the foliation $\mathcal{F}\left(e^{2 i \theta} \omega^{2}\right)$ has closed leaves. The parabolic element $A_{i}$ can be computed in terms of $c_{i}$ by decomposing $X$ into annuli and taking the least common multiple of their reciprocal moduli, as in Lemma 9.7.

If the chain of axes $\left\langle\gamma_{i}\right\rangle$ ever closes or crosses itself, it then bounds a polygon of finite volume $F \subset \mathbb{H}$. Then the reflections found so far generate a lattice $\Gamma$, and $\Gamma \cap \mathrm{SL}_{2}(\mathbb{R})$ gives an explicit subgroup of finite index in $\operatorname{SL}(X, \omega)$. In this case the Teichmüller curve $V$ has genus 0 , since it is covered by the double of $F$.

We apply the algorithm to the surface $(X, \omega)$ associated to the polygon $P(a, a)$ as follows. Because the Swiss cross is symmetric under horizontal, vertical and diagonal Euclidean reflections, the hyperbolic reflections $R_{0}=\left(\begin{array}{cc}1 & 0 \\ 0 & -1\end{array}\right)$ and $\widetilde{R}_{0}=$ $\left(\begin{array}{ll}0 & 1 \\ 1 & 0\end{array}\right)$ both belong to $\operatorname{GL}(X, \omega)$. Starting with $R_{0}, \widetilde{R}_{0}$, and the corresponding cusps $c_{0}=\infty, \widetilde{c}_{0}=1$, we generate two chains of axes as described above. If these chains happen to meet or cross, we obtain the desired lattice in $\operatorname{SL}(X, \omega)$.

Concretely, starting with $\left(R_{0}, c_{0}\right)$, the algorithm proceeds as follows. The cusp $c_{0}=\infty$ determines a decomposition of $X$ into horizontal annuli of moduli $a-1$ and $1 / a$, corresponding to the two rectangles that are stacked to make $P(a, a)$. Let $t=\operatorname{lcm}\left(a,(a-1)^{-1}\right)$. By Lemma 9.7, the shear $A_{0}=\left(\begin{array}{ll}1 & t \\ 0 & 1\end{array}\right)$ belongs to $\operatorname{GL}(X, \omega)$, as does the reflection $R_{1}=A_{0} R_{0}$ through the line $\operatorname{Re}(z)=t / 2$. From $R_{1}$ we obtain the next cusp, $c_{1}=t / 2$, and so on.

A sampler. Figure [5] shows the reflection groups found in this way for the values of $d$ given in Theorem 9.8 .

When $d=5$, we obtain the $(2,5, \infty)$ triangle group. In this case $a=\gamma$ is the golden mean, so $1 / a=a-1$ and $R_{1}$ is reflection through the line $\operatorname{Re}(z)=\gamma / 2$. The axes $\operatorname{Re}(z)=0, \operatorname{Re}(z)=\gamma / 2$ and $|z|=1$ of $R_{0}, R_{1}$ and $\widetilde{R}_{0}$ bound a $(2,5, \infty)$ triangle $F \subset \mathbb{H}$, providing a fundamental domain for the group generate by these three reflections.

When $d=17$, the fundamental domain $F$ is an ideal quadrilateral with vertices $(i, 1,(1+\sqrt{17}) / 4, \infty)$.

The algorithm works best when $d=1 \bmod 4$, since then the cusp $c_{1}=(1+\sqrt{d}) / 4$ adjacent to infinity is fairly close to 1 . For $d \neq 1 \bmod 4$, the corresponding cusp is at $(1+\sqrt{d}) / 2$ or at $1+\sqrt{d}$.

It would be interesting to design an algorithm to compute the full group $\operatorname{SL}(X, \omega)$, especially when $\mathbb{H} / \mathrm{SL}(X, \omega)$ has higher genus. 


\section{Holomorphic Galois CONJugation}

Let $V \subset \Sigma \subset \mathcal{A}_{2}$ be a Teichmüller curve on a Hilbert modular surface. In this final section we observe that $V$ is covered by the graph of a remarkable holomorphic map $F: \mathbb{H} \rightarrow \mathbb{H}$ intertwining the action of a discrete group $G \subset \mathrm{SL}_{2}(\mathbb{R})$ and its indiscrete Galois conjugate $G^{\prime}$.
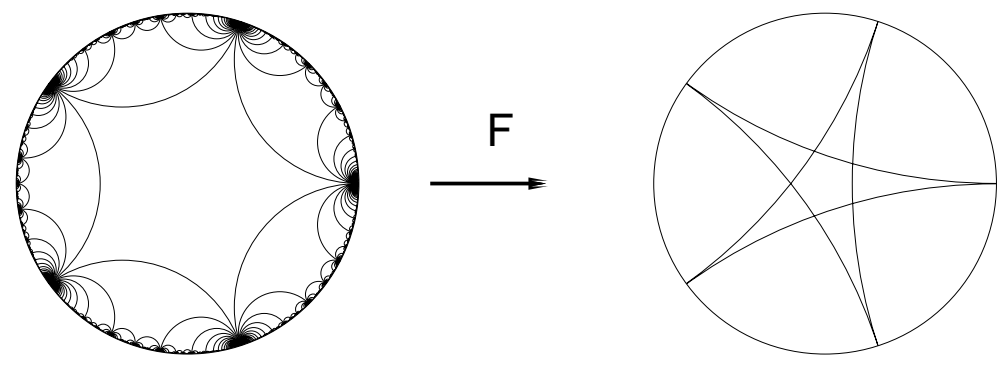

Figure 6. A holomorphic map from an ideal pentagon to an ideal star.

Curves on surfaces. Let $f: V \rightarrow \mathcal{M}_{2}$ be a Teichmüller curve generated by $\left(X, \omega^{2}\right)$. Assume that $\operatorname{SL}(X, \omega)$ has real quadratic trace field $K \subset \mathbb{R}$. Let $k \mapsto k^{\prime}$ denote the Galois involution of $K / \mathbb{Q}$, sending $a+b \sqrt{d}$ to $a-b \sqrt{d}$, and let $g \mapsto g^{\prime}$ denote the corresponding involution on $\mathrm{SL}_{2}(K)$.

We have seen that $\operatorname{Jac}\left(X_{t}\right), t=f(t)$, admits real multiplication by $K$ for all $t$. Let $\Sigma=\mathbb{H} / \Gamma, \Gamma \subset \mathrm{SL}_{2}(K)$, be the Hilbert modular surface parameterizing all Abelian varieties with the given action of $K$, as in $\sqrt{6}$. Then we obtain a commutative diagram

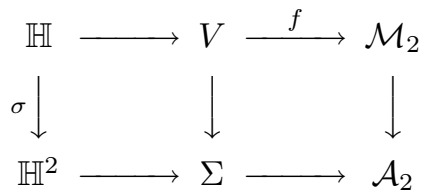

as in Corollary 7.3 ,

If we make a change of basepoint for $V$, by replacing $(X, \omega)$ with $A \cdot(X, \omega)$, $A \in \mathrm{SL}_{2}(\mathbb{R})$, then $f(V) \subset \mathcal{M}_{2}$ does not change and $\mathrm{GL}(X, \omega)$ varies only by conjugation by $A$. The main observation of this section is:

Theorem 10.1. After a suitable change of basepoint, we have $\mathrm{GL}(X, \omega) \subset \mathrm{GL}_{2}(K)$ and $\sigma(t)=(t, F(t))$, where $F: \mathbb{H} \rightarrow \mathbb{H}$ satisfies

$$
F(g \cdot t)=g^{\prime} \cdot F(t)
$$

for all $g \in \mathrm{GL}(X, \omega)$. The holomorphic map $F$ is a contraction for the hyperbolic metric.

The Hilbert modular surface $\Sigma$ is abundantly populated by modular curves (Shimura curves), parameterizing Abelian varieties with an action of a quaternion algebra $\mathrm{VG}, \S \mathrm{V}]$. However these curves are covered by the graphs of isometries, so we have:

Corollary 10.2. The Teichmüller curve $V \subset \Sigma$ is not a modular curve. 
Since $F$ is a contraction, the spectral radius of every hyperbolic element $g \in$ $\mathrm{GL}(X, \omega)$ must decrease under Galois conjugation. This shows:

Corollary 10.3. The trace of every hyperbolic element in $\operatorname{GL}(X, \omega)$ is irrational.

Example: $K=\mathbb{Q}(\sqrt{5})$. Here is an example where the equivariant map $F$ provided by Theorem 10.1 can be seen directly.

Consider the Teichmüller curve generated by the regular pentagon or equivalently by the golden table. Then $G=\mathrm{GL}(X, \omega)$ is the group generated by reflections in the edges of the $(2,5, \infty)$ triangle, as we saw in 9 .

Changing coordinates from the upper halfplane to the unit disk $\Delta$, we can assume $G$ contains the reflections in the edges of the ideal pentagon $P$ with vertices at the 5 th roots of unity. Under the action of $\operatorname{Gal}(K / \mathbb{Q})$, the edge joining $\left(\zeta^{i}, \zeta^{i+1}\right)$ is sent to the edge joining $\left(\zeta^{2 i}, \zeta^{2 i+2}\right)$. Thus $G^{\prime}$ is the indiscrete group generated by the reflections in the edges of an ideal star $P^{\prime}$ (see Figure 6).

There is a unique holomorphic map $F: P \rightarrow P^{\prime}$ sending edges to corresponding edges. This map has a simple critical point at the origin. By repeated Schwarz reflection, $F$ can be analytically continued to the entire disk. The continuation $F: \Delta \rightarrow \Delta$ intertwines the actions of $G$ and $G^{\prime}$, and its graph covers the Teichmüller curve generated by the regular pentagon. In fact $F$ is determined by its equivariance properties, since its boundary values depend only on the isomorphism $G \cong G^{\prime}$.

Proof of Theorem 10.1. Let $\left(X_{t}, \omega_{t}\right)=A_{t} \cdot(X, \omega)$, so $X_{t}=f(t)$. Then the map $\sigma: \mathbb{H} \rightarrow \mathbb{H}^{2}$ in the diagram (10.1) has the form

$$
\sigma(t)=\left(\sigma_{11}(t), \sigma_{22}(t)\right)=\left(\frac{\int_{b_{1}^{\prime}} \eta_{1}(t)}{\int_{a_{1}^{\prime}} \eta_{1}(t)}, \frac{\int_{b_{2}^{\prime}} \eta_{2}(t)}{\int_{a_{2}^{\prime}} \eta_{2}(t)}\right),
$$

where $\left(a_{i}^{\prime}, b_{i}^{\prime}\right), i=1,2$, is a symplectic basis for $H_{1}\left(Z_{2}, \mathbb{R}\right)$ adapted to the action of $K$ and $\left(\eta_{1}(t), \eta_{2}(t)\right)$ is a dual eigenbasis for $\Omega\left(X_{t}\right)$.

Interchanging coordinates if necessary, we can assume $\eta_{1}(t)=\omega_{t}$. By Theorem 4.2, the map $\sigma_{11}: \mathbb{H} \rightarrow \mathbb{H}$ is an isometry. Therefore after a change of basepoint we can assume $\sigma_{11}(t)=t$ and write $\sigma(t)=(t, F(t))$. By Theorem 4.2 $F(t)=\sigma_{22}(t)$ is a contraction for the hyperbolic metric.

The action of the $\mathrm{Aff}^{+}(X, \omega)$ on $H_{1}(X, \mathbb{Q}) \cong K^{2}$ commutes with the action of $K$, so we have a natural inclusion

$$
E: \operatorname{Aff}^{+}(X, \omega) \hookrightarrow \mathrm{SL}_{2}(K) .
$$

Similarly the derivative gives a natural map

$$
D: \operatorname{Aff}^{+}(X, \omega) \rightarrow \mathrm{SL}(X, \omega) \subset \mathrm{SL}_{2}(\mathbb{R}) .
$$

The corresponding actions on the universal covers of $V$ and $\Sigma$ are compatible; that is, if $A=D \phi$ and $g=E \phi$, then

$$
\sigma(A \cdot t)=g \cdot \sigma(t)=\left(g \cdot t, g^{\prime} \cdot F(t)\right) .
$$

But in fact $A=g$ (under the inclusion $\mathrm{SL}_{2}(K) \subset \mathrm{SL}_{2}(\mathbb{R})$ ), since both matrices describe the action of $\phi$ on the space of 1 -forms with basis $\{\operatorname{Re} \omega, \operatorname{Im} \omega\}$ (compare equation (3.3) ). Therefore $D$ is an isomorphism, we have $\operatorname{SL}(X, \omega) \subset \mathrm{SL}_{2}(K)$, and $F(g \cdot t)=g^{\prime} \cdot F(t)$ for all $g \in \operatorname{SL}(X, \omega)$.

Note. The above example of a curve $V \cong \mathbb{H} / \Gamma(2,5, \infty)$ on the Hilbert modular surface for $\mathrm{SL}_{2}\left(\mathcal{O}_{\mathbb{Q}(\sqrt{5})}\right)$ is also discussed in [CW] and [Sch]. See [SW] for other 
work on "modular embeddings" of Fuchsian groups. Another proof of Corollary 10.3 is given in [Mc, Cor. 9.6].

\section{ACKNOWLEDGMENTS}

I would like to thank B. Gross and H. Masur for useful conversations.

\section{ADDED AFTER POSTING}

Just before the statement of Proposition 6.2, the sentence ending:

by $\widetilde{f}(t)=T \cdot \delta(t)$.

should read:

$$
\text { by } \widetilde{f}(t)=T \cdot \delta(t) \cdot T^{-1} \text {. }
$$

\section{REFERENCES}

[Ah] L. Ahlfors. The complex analytic structure of the space of closed Riemann surfaces. In Analytic Functions, pages 45-66. Princeton Univ. Press, 1960. MR 23:A1798

[Ca] K. Calta. Veech surfaces and complete periodicity in genus 2. Preprint, 5/2002.

$[\mathrm{CV}]$ C. Ciliberto and G. van der Geer. Subvarieties of the moduli space of curves parametrizing Jacobians with nontrivial endomorphisms. Amer. J. Math. 114(1992), 551-570. MR 93d:14044

[CVT] C. Ciliberto, G. van der Geer, and M. Teixidor i Bigas. On the number of parameters of curves whose Jacobians possess nontrivial endomorphisms. J. Algebraic Geom. 1(1992), 215-229. MR 93c:14025

[CW] P. Cohen and J. Wolfart. Modular embeddings for some non-arithmetic Fuchsian groups. Acta Arith. 56(1990), 93-110. MR 92d:11039

[CFS] I. P. Cornfeld, S. V. Fomin, and Ya. G. Sinai. Ergodic Theory. Springer-Verlag, 1982. MR 87f:28019

[EO] A. Eskin and A. Okounkov. Asymptotics of numbers of branched coverings of a torus and volumes of moduli spaces of holomorphic differentials. Invent. Math. 145(2001), 59-103. MR 2002g:32018

[FLP] A. Fathi, F. Laudenbach, and V. Poénaru. Travaux de Thurston sur les surfaces. Astérisque, volume 66-67, 1979.

[GR] B. H. Gross and D. E. Rohrlich. Some results on the Mordell-Weil group of the Jacobian of the Fermat curve. Invent. Math. 44(1978), 201-224. MR 58:10911

[GJ] E. Gutkin and C. Judge. Affine mappings of translation surfaces: geometry and arithmetic. Duke Math. J. 103(2000), 191-213. MR 2001h:37071

[HG] F. Hirzebruch and G. van der Geer. Lectures on Hilbert Modular Surfaces. Les Presses de l'Université de Montréal, 1981. MR 83i:10037

[KS] R. Kenyon and J. Smillie. Billiards on rational-angled triangles. Comment. Math. Helv. 75(2000), 65-108. MR 2001e:37046

[KMS] S. Kerckhoff, H. Masur, and J. Smillie. Ergodicity of billiard flows and quadratic differentials. Ann. of Math. 124(1986), 293-311. MR 88f:58122

[Ko] M. Kontsevich. Lyapunov exponents and Hodge theory. In The Mathematical Beauty of Physics (Saclay, 1996), pages 318-332. World Sci. Publishing, 1997. MR 99b:58147

[KZ] M. Kontsevich and A. Zorich. Connected components of the moduli spaces of Abelian differentials with prescribed singularities. Preprint, 2002.

[Kra] I. Kra. The Carathéodory metric on abelian Teichmüller disks. J. Analyse Math. 40(1981), 129-143. MR 83m:32027

[Li] D. Lind. The entropies of topological Markov shifts and a related class of algebraic integers. Ergod. Th. ES Dynam. Sys. 4(1984), 283-300. MR 86c:58092

[Mas1] H. Masur. Transitivity properties of the horocyclic and geodesic flows on moduli space. $J$. Analyse Math. 39(1981), 1-10. MR 82k:30047 
[Mas2] H. Masur. Lower bounds for the number of saddle connections and closed trajectories of a quadratic differential. In Holomorphic Functions and Moduli I, pages 215-228. SpringerVerlag: MSRI publications volume 10, 1988. MR 90e:30046

[Mas3] H. Masur. Hausdorff dimension of the set of nonergodic foliations of a quadratic differential. Duke Math. J. 66(1992), 387-442. MR 93f:30045

[MT] H. Masur and S. Tabachnikov. Rational billiards and flat structures. In Handbook of Dynamical Systems, Vol. 1A, pages 1015-1089. North-Holland, 2002.

[Mc] C. McMullen. Teichmüller geodesics of infinite complexity. To appear, Acta Math.

[Pen] R. Penner. Bounds on least dilatations. Proc. Amer. Math. Soc. 113(1991), 443-450. MR 91m:57010

[Pu] J.-C. Puchta. On triangular billiards. Comment. Math. Helv. 76(2001), 501-505. MR 2002f: 37060

[Rap] M. Rapoport. Compactifications de l'espace de modules de Hilbert-Blumenthal. Compositio Math. 36(1978), 255-335. MR 80j:14009

[Roy] H. L. Royden. Invariant metrics on Teichmüller space. In Contributions to Analysis, pages 393-399. Academic Press, 1974. MR 51:13290

[SW] P. S. Schaller and J. Wolfart. Semi-arithmetic Fuchsian groups and modular embeddings. J. London Math. Soc. 61(2000), 13-24. MR 2001a:11071

[Sch] T. A. Schmidt. Klein's cubic surface and a 'non-arithmetic' curve. Math. Ann. 309(1997), 533-539. MR 98m:11036

[Tab] S. Tabachnikov. Billiards. Société Mathématique de France, 1995.

[vG] G. van der Geer. Hilbert Modular Surfaces. Springer-Verlag, 1987. MR 89c:11073

[V1] W. Veech. Teichmüller curves in moduli space, Eisenstein series and an application to triangular billiards. Invent. Math. 97(1989), 553-583. MR 91h:58083a

[V2] W. Veech. Moduli spaces of quadratic differentials. J. Analyse Math. 55(1990), 117-171. MR 92e:32014

[V3] W. Veech. The billiard in a regular polygon. Geom. Funct. Anal. 2(1992), 341-379. MR 94a:11074

[V4] W. Veech. Geometric realizations of hyperelliptic curves. In Algorithms, Fractals and Dynamics (Okayama/Kyoto, 1992), pages 217-226. Plenum Publishing, 1995. MR 98f:14022

[Vo] Ya. B. Vorobets. Plane structures and billiards in rational polygons: the Veech alternative. Russian Math. Surveys 51(1996), 779-817. MR 97j:58092

[Wa] C. C. Ward. Calculation of Fuchsian groups associated to billiards in a rational triangle. Ergod. Th. \& Dynam. Sys. 18(1998), 1019-1042. MR 2000b:30065

Mathematics Department, Harvard University, 1 Oxford Street, Cambridge, MasSACHUSETTS 02138-2901 